\title{
El comercio gaditano a fines del siglo XIX: Análisis de un año de actividad portuaria
}

\section{GLORIA ESPIGADO TOCINO}

No descubrimos nada nuevo si apuntamos que el pasado económico de Cádiz está vinculado a la actividad comercial que desarrolla su puerto. Enseguida, la fácil asociación de ideas entre su muelle y los viajes a ultramar nos asalta y nos llegamos a imaginar sin dificultad la ola de prosperidad que la engrandeció en el siglo XVIII. Igualmente, tan intuitivo como lo anterior, es rememorar su decadencia a raíz de la pérdida de los privilegios monopolísticos en 1778 , pero, sobre todo, resulta complicado cuestionar o relativizar la profunda sima que solemos unir a la emancipación colonial producida en los años veinte del ochocientos. Esta impresión general que hemos descrito puede deberse a la existencia de importantes trabajos que analizan pormenorizada y brillantemente la evolución de su comercio en el ciclo de bonanza que comienza con el traslado de la Casa de Contratación de Sevilla a Cádiz en 1717, nos referimos al magnífico trabajo del profesor GarcíaBaquero, Cádiz y el Atlántico (1), mientras habrá que reconocer que la imagen de decadencia se nutre, más que de trabajos rigurosos sobre la realidad comercial del XIX, de hipótesis todavía no comprobadas científica y empíricamente. Es Nicolás Sánchez-Albornoz en "Cádiz, ciudad revolucionaria en la encrucijada económica" (2), el primero que intuye otra realidad y rompe con esta imagen tópica de infortunio comercial.

(1) García-Baquero ANTONIO, Cádiz y el Atlántico (1717-1778) (El comercio colonial español bajo el monopolio gaditano). Publicaciones de la Escuela de Estudios Hispano-Americanos de Sevilla, 1976, y también de este autor Comercio colonial y guerras revolucionarias. La decadencia económica de Cádiz a raíz de la emancipación americana. Publicaciones de la Escuela de Estudio Hispano-Americanos de Sevilla, 1972.

(2) En Lida Clara E. y Zabala Iris $M^{\mathrm{a}}$, La Revolución de 1868, Historia, Pensamiento y Literatura. Las Américas Publishing Company, New York, 1970, pp. 80-108. 
Ayudándose de las cifras consulares francesas, llega a demostrar que los años centrales del XIX transpiran prosperidad y una buena marcha en los negocios puesta de relieve por el progresivo incremento del valor de las transacciones. Nos advierte, acertadamente, de un posible cambio de orientación de los intercambios gaditanos, crecientemente vinculados a Europa, mientras permanecen moderadamente relacionados con el espacio americano y concretamente con las colonias caribeñas, que no se pierden sino al finalizar la centuria. Es posible que Cádiz, por aquel entonces, haya dejado de ser el emporio que canalizaba a manos llenas el oro y la plata americanas, sin embargo, existen indicadores suficientes como para poner en tela de juicio la visión tópica de declive secular sistemáticamente atribuida al pasado siglo, que bastan para introducir, dentro del asumido trend de línea descendente que hasta hoy nos llega, una visión cíclica más realista y conveniente para entender nuestro ayer económico. El terreno es virgen y hay mucho por hacer, por el momento, y reconociendo las limitaciones a que está sujeta la investigación que aquí introducimos, nos damos por satisfechos si contribuimos a despertar el interés sobre el estado material de esta ciudad en la pasada centuria, sombra y cruz, reverso del refulgente XVIII que, por ello mismo, resulta ser injustamente tratada.

Siendo estrictos, también nosotros recalamos indirectamente sobre estas cuestiones. La exigencia de dibujar con precisión la coyuntura económica gaditana en el transcurso de la Primera Repúbli$\mathrm{ca}$, tema de nuestra recientemente concluida Tesis doctoral, nos obligó a adentrarnos en todos aquellos parámetros indicativos del pulso económico de una localidad como Cádiz. El estado de las finanzas, de las manufacturas, de los precios, de los salarios, del paro, etc., y, especialmente, su comportamiento como centro tradicional de importación y exportación de mercancías, fueron analizados. Como suele suceder, toda indagación sincrónica en el tiempo histórico termina por rendirse ante la evidente necesidad de insertar datos tan precisos en un contexto más amplio que dé sentido y ayude a explicar el punto en que nos movemos; esto es lo que nos sucedió a nosotros, y fruto de esa exigencia son los cuadros que seguidamente introducimos y que, elaborados a partir del movimiento portuario registrado anualmente en el Boletín Oficial de la Provincia de Cádiz, reflejan tanto el número de buques entrados como el tonelaje desplazado por los mismos, haciendo en ambos casos alusión al origen nacional o extranjero del pabellón que les corresponde, desde mediados hasta el final de la centuria. 
Gráfico 1: Entradas de buques mercantes en el puerto gaditano

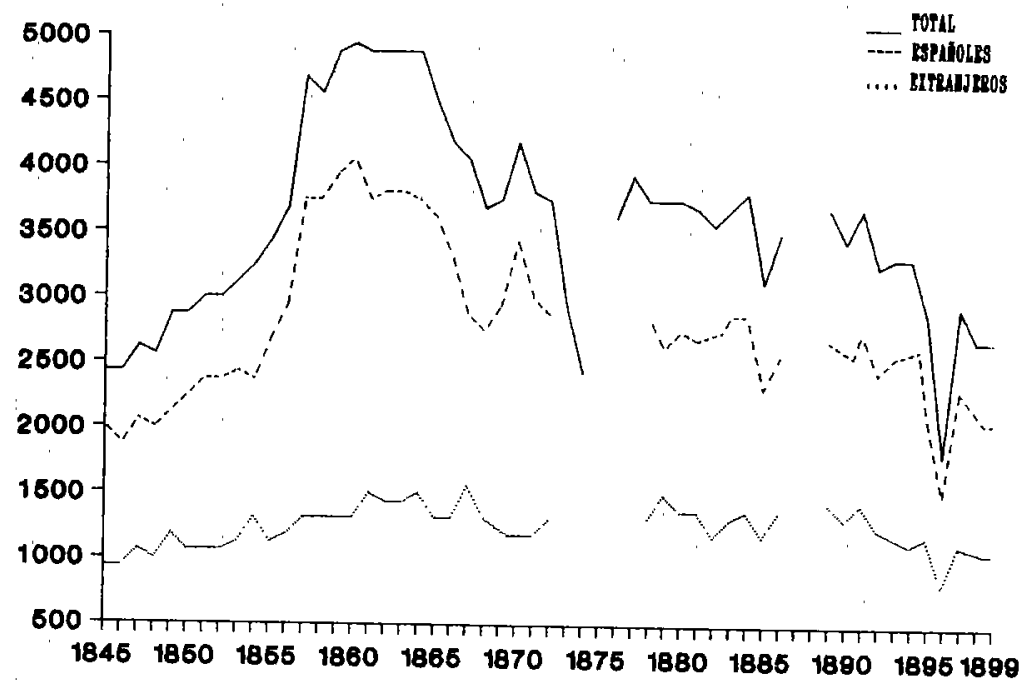

Fuente: B.O.P. de Cádiz, años 1845-1872; C.C.C., Cádiz, vol. 113, despacho 332, de 6 de abril de 1875, p. 140; Guía de José Rosetty, años 1876-1900.

Gráfico 2: Tonelaje de los barcos mercantes españoles entrados en el puerto de Cádiz (en miles de T.M.)

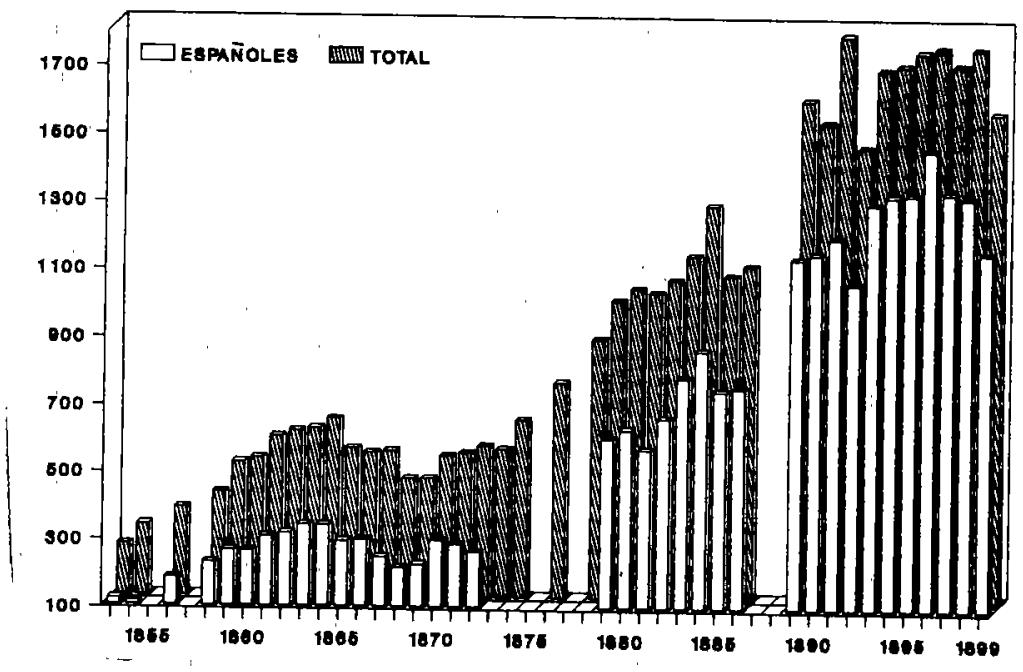


Resulta fácil comprobar, observando la gráfica que reproduce el movimiento de buques entrados en el puerto (gráfico $n^{-0} 1$ ), que la curva final señala perfectamente el aumento, en principio gradual, de las transacciones comerciales a medida que avanzamos hacia mediados de siglo, que se convierte en espectacular, casi vertical, a partir de 1853-1854. Siguen, a continuación, unos años de prosperidad en que se mantienen, año a año, los valores máximos del siglo hasta que, llegando 1864, año fatídico, se experimenta un descenso tan brusco como fue el ascenso anterior. En cuanto al tonelaje de esos barcos registrados en esos años (gráficas 2 y 3), factor más significativo para calificar la actividad del puerto comercial, es evidente que confirma, paso a paso, el proceso anterior de subida y bajada descrita (en 1868, año en que se toca fondo, el número de barcos entrados experimentó un descenso de casi un $24 \%$ respecto a 1864 , del mismo modo el tonelaje pesado bajó hasta un $28 \%$ ).

Si analizamos pormenorizadamente la procedencia de los diferentes barcos y su evolución cuantitativa en relación a la etapa 1860-1864 (indice 100 del gráfico $\mathrm{n}^{\mathbf{0}}$ 4), concluiremos que tras el grave bache producido por la crisis se experimenta una decidida mejoría en el primer año cumplido de la Revolución que, sin embargo, en la mayoría de los casos no implica la superación del índice 100 tomado como referencia, y que, igualmente, no se prolonga más allá de 1870-71. El comercio colonial, representado por los barcos venidos de Asia y América, parece condenado a no recuperar los valores que antaño situaban a Cádiz en una posición privilegiada. No menos significativa es la caída casi en vertical en 1872 de las manufacturas extranjeras traídas por barcos nacionales. El retraimiento del consumo viene marcado, de la misma manera, por la reducción y estancamiento del comercio de cabotaje realizado por barcos de pequeño tonelaje (faluchos, laúdes, barcas...) que, procedentes de puertos cercanos (pueblos de Huelva, de Sevilla o de Málaga...), proporcionan pescado, frutos, huevos, ladrillos, etc... En cambio, parece notable el crecimiento de la aportación de otros puertos de la geografía nacional que requieren embarcaciones de mayor envergadura para el transporte de sus mercancías. Podemos convenir, analizando finalmente la entrada de buques que nos llegan del extranjero, que su concurso parece sustraerse a la tónica general marcada por la crisis de 1864 en los demás casos y que, además, con toda probabilidad, la responsabilidad final del incremento del valor del comercio que veremos para la etapa que coincide con el Sexenio democrático recae en el espectacular aumento de buques ex(tanjeros (ingleses, franceses e italianos, fundamentalmente) que en 
Gráfico 3: Tonelaje de los barcos mercantes extranjeros entrados en el puerto de Cádiz (en miles de T.M.)

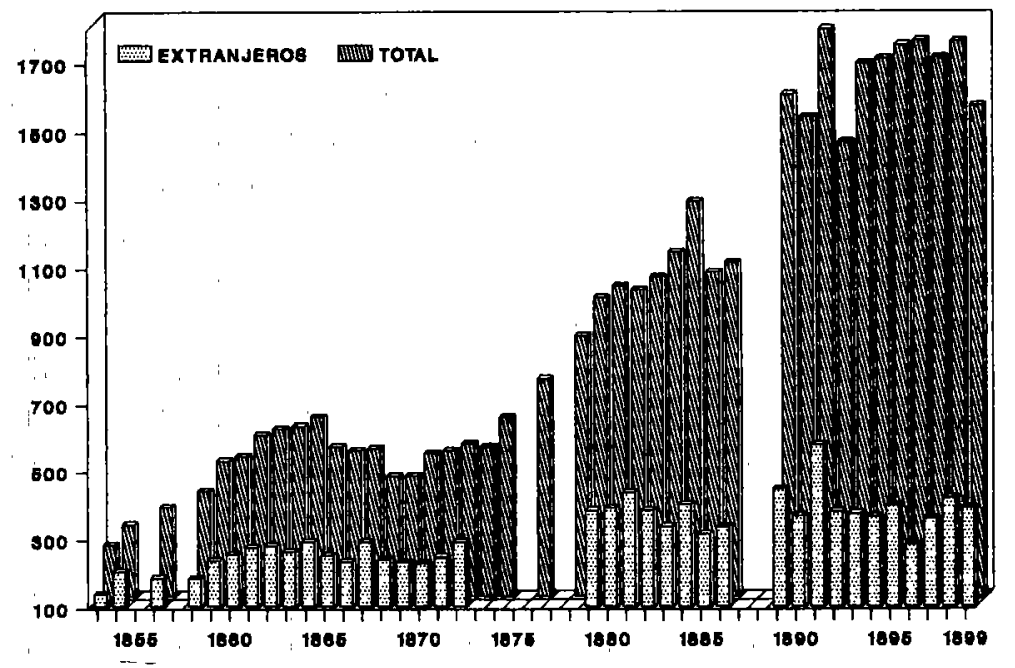

Fuente: B.O.P. de Cádiz, años 1853-1872; C.C.C., Cádiz, vol. 113, despacho 332, de 6 de abril de 1875, p. 140; Guía de José Rosetty, años 1876-1900.

Gráfico 4: Variación del tonelaje de los barcos mercantes entrados en el puerto de Cádiz según su procedencia respecto a 1860-1864 (índice 100).

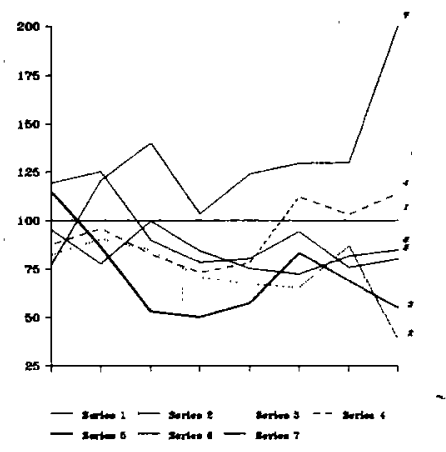

Serie $1,1860-64=100$

Serie 2, Españoles procedentes de las colonias. Serie 3, Españoles procedentes del extranjero. Serie 4, Españoles cabotaje (+20 TM).
Serie 5, Españoles cabotaje (-20 TM).

Serie 6, Extranjeros con carga. Serie 7, Extranjeros en lastre.

Fuente: B.O.P. de Cádiz, años 1860-1872. 
lastre, es decir, sin carga previa alguna, se acercan a Cádiz para llenar sus bodegas exclusivamente de sal y/o vino para continuar rumbo a América y Europa principalmente.

Nuestra inquietud por conocer más a fondo lo que encerraban estas cifras globales sobre el tráfico marítimo nos llevó a interesarnos por la realidad, barco a barco, que se correspondía con la actividad portuaria de ese año. El registro del movimiento habido diariamente que contempla la prensa local podía ser una buena forma de informarnos al respecto. Partiendo de esta idea, fue utilizado El Comercio por la oportunidad de disponer de una serie completa que no ofreciese lagunas, al conservarse íntegramente la publicación correspondiente a 1873. Los datos recabados fueron dispuestos en dos bases de datos que apreciaban, por un lado, el tráfico de entrada, con 3.100 registros, y, por otra, el de salida, con 1.530 casos (la disparidad entre ambas cifras se debe a que en el recuento oficial no son consideradas las salidas que llevan a cabo las embarcaciones menores, y sí, en cambio, lo son en sus entradas). Los campos abiertos eran comunes a ambas bases y pretendian revelar, en primer lugar, el nombre y el tipo de embarcación, así como la nacionalidad de su pabellón. Tres campos fueron dedicados a registrar su carga, en previsión de que ésta fuese múltiple, y otros tantos campos para los lugares de procedencia o destino, según el caso, que se citasen. Finalmente, y sólo con la base de datos que reflejaba las entradas, podríamos conocer a nombre de quién estaban hechas las consignaciones de los barcos, por lo que se introdujo un campo con el fin de averiguar posibles casos de especialización en cargas o rutas de estos comerciantes. La oportunidad de establecer relaciones entre los diferentes campos no ha sido desaprovechada, como tampoco ha sido despreciada la posibilidad de acudir a otro tipo de fuentes complementarias que serán presentadas a su debido tiempo, puesto que ya es hora de que nos adentremos, sin más dilaciones, a desentrañar lo que consideramos constituyen los aspectos esenciales para entender una faceta, quizá la más importante, de la función económica que le corresponde desempeñar a Cádiz en el último cuarto del siglo pasado.

\section{LOS PAISES}

Si analizamos la procedencia de los buques que en Cádiz tocan puerto, resulta ser mayoritario lógicamente el pabellón nacional, con más del $73 \%$ de las entradas y un $56 \%$ de las salidas. Recordemos que la diferencia notable entre las cifras totales de barcos españoles entrados y salidos se debe a la ausencia de faluchos, místicos y laúdes, en 
definitiva, de embarcaciones menores, en los registros oficiales de salida. Considerando que estos tipos se encuentran representados en las entradas con, aproximadamente, unas 1.500 unidades, es fácil deducir que lós 855 buques contabilizados en las salidas representan el tráfico de gran tonelaje realizado por los mercantes nacionales.

Gráfica 5: Relación entre buques nacionales y extranjeros en las entradas y salidas registradas en 1873

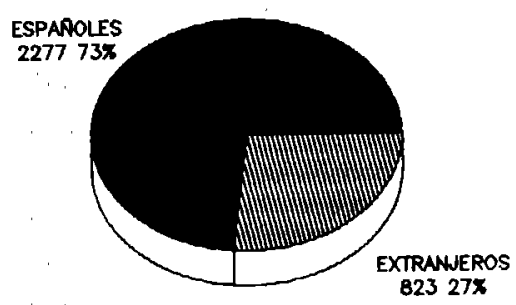

ENTRADAS

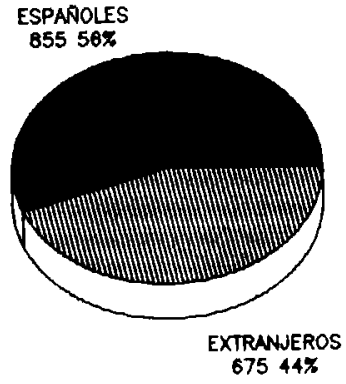

SALIDAS

Respecto al comercio efectuado en buques extranjeros es más que notable la participación inglesa, con algo más de 300 barcos entrados o salidos del puerto. Afinando sobre la naturaleza de su intercambio, son aproximadamente un tercio los que llegan sin carga, en lastre, a la ciudad y otro tanto lo hacen con carbón en sus bodegas. El resto del transporte británico arribado a estas costas andaluzas se compone de mineral de hierro en bruto o previamente laborado en tuberías, duelas de madera americana para la construcción de toneles y bacalao pescado en las costas de Terranova. También parece haber un tráfico regular de pasajeros y mercancías entre Cádiz y Gibraltar de responsabilidad británica. En cuanto a lo que los ingleses se llevan de Cádiz, la sorpresa salta al verificar que el vino ocupa el segundo lugar en importancia con 85 buques, tras la sal, que es, a no dudar, la mercancía reina que viene a buscar preferentemente la mayor parte del transporte realizado bajo pabellón extranjero (son 138 los navíos ingleses que parten con sal en sus bodegas rumbo a las pesquerías de Terranova). Si con vino y/o sal llenan sus bodegas el $73 \%$ de los navíos ingleses, al menos un $15 \%$ opta por salir de vacío, en lastre, de las costas andaluzas, 45 embarcaciones que, en su mayoría, se dirigen a Huelva para cargar mineral, completando el típico esquema triangular del comercio británico.

Los franceses, segundos en importancia y a considerable distancia del tráfico inglés, puesto que no suponen siquiera la mitad de éste, no 
parecen llamados a satisfacer demanda alguna, al entrar lastrada casi la mitad de su flota ( 42 barcos). De sus posesiones en Africa suroriental, islas de Reunión y Mauricio, Francia nos trae 12 barcos cargados de azúcar de camino hacia Marsella, Nantes, etc. Un número importante representado por 33 barcos no llega a especificar la carga y 18 declaran llevar "varios efectos" o "carga general". Sólo queda por comentar los 8 que traen carbón, para tener casi al completo la flota gala. Salen, en número de 45 , de igual modo, con sal para diferentes puerto americanos, principalmente para las salazones de pescado de Terranova y de ganado de Montevideo.

El tercer pais en importancia por el volumen de su comercio de entrada, Portugal, precisa una observación que explique su posición en la cola cuando consideramos los viajes de regreso. Las 111 unidades que nos visitan se refieren exclusivamente al aporte de pescado que desde Fuseta y Olhao (57 y 39 viajes, respectivamente), puertos próximos del país vecino, nos llega en pequeñas embarcaciones como son los místicos, el tradicional caique portugués y las, también lusitanas, canoas. En el primer tipo se hacen 53 llegadas, en el segundo 41 y en el último 7.

La diferencia en el número de entradas y salidas de los mercantes italianos en favor de las segundas, obviamente responde a un error, por omisión, en el registro de las llegadas. No sorprendería en Cádiz que de los 72 buques italianos que nos visitan, la mayor parte, 48 exactamente, nos lleguen desde Génova, perpetuándose así los viejos lazos comerciales que en la Edad Moderna unían a estas dos ciudades. Algo más de la mitad de la flota de nuestros tradicionales intermediarios con el comercio indiano ( 61 buques de las 79 salidas registradas), navega con el único propósito de llevar el fruto blanco del mar hacia los puertos de Montevideo, Río de la Plata y Buenos Aires. Tan sólo quedan algunos que conducen vino a Nueva York. Finalmente, también entra dentro de la lógica comprobar como lo tres aportes de mármol que recibimos han sido estibados en la ciudad de Génova.

El muelle estadounidense con el que se mantiene mayor número de contactos es el de New York, con 27 viajes de ida y 37 de vuelta. Los norteamericanos, en un alto porcentaje, traen duelas con que construir los barriles que almacenarán el vino jerezano, 28 de los 41 buques descargados a su llegada (3). El tabaco y el petróleo son

(3) Manuel Ma. González Gordon, en su libro Jerez, Xerez, Sberish. Noticias sobre el origen de esta ciudad, su bistoria y su vino, Jerez de la Frontera, 1970, pp. 442450 , nos habla del aprecio que se tenía al roble americano para la fabricación de los recipientes donde iban a envejecer los caldos de la tierra. 
secundarios en este comercio, pero constituyen otras partidas a destacar. Por otra parte, nada nuevo para el viaje de regreso, la sal aparece como el bien primordial apetecido también por los norteamericanos, 33 barcos para New York, Boston, Gloucester, Filadelfia o Nueva Orleans, mientras que tan sólo 5 saldrán con vino hacia el Nuevo Continente.

Los alemanes no parecen tener muy especializadas ni sus entradas ni sus salidas, aunque podemos decir que suelen llegar lastrados o con aguardiente desde Hamburgo y con carbón inglés, zarpando principalmente con vino hacia la zona del Báltico, con dirección a Hamburgo, Copenhague, San Petersburgo, etc.

Nos hemos saltado intencionadamente en nuestro comentario el aporte noruego, puesto que conjuntamente con el holandés, ruso, sueco y danés, componen un bloque homogéneo por cuanto se refiere a la naturaleza de su comercio. Noruegos, holandeses y daneses funcionan fundamentalmente como intermediarios, haciendo escala en puertos británicos para traernos carbón (15 noruegos, 5 holandeses, y 5 daneses). Los noruegos también vienen del Báltico con madera, en nueve ocasiones, y bacalao en cuatro; curiosamente, dos llegan con hielo. Estos países cargan en sus bodegas sal y/o vino, bien para los puertos tradicionales norte o sudamericanos, ya vistos, o para sus países de origen.

Rusia y Suecia están especializadas en el comercio maderero, tomando de regreso el vino y la sal que conducirán hacia los mismos destinos que el grupo de países anteriormente mencionado, siendo el puerto de Copenhague especialmente visitado.

El tráfico belga, griego o austriaco, al final de toda la relación, entra por su escasa relevancia dentro de lo anecdótico. La fragata belga viene con cueros desde Buenos Aires y zarpa con la misma carga rumbo a Barcelona. Los griegos traen avellanas desde Río Muni y van de paso hacia Marsella; por fin, los dos austriacos, en lastre y con cueros desde Venecia y Buenos Aires, respectivamente, marchan con sal hacia Montevideo y Boston.

Procede insertar este comentario sobre las diferentes naciones con las que se mantienen contactos comerciales hacia 1873 en un contexto más amplio que nos descubra las constantes en el reparto del comercio entre los diferentes pabellones extranjeros. El período elegido es el inmediatamente anterior a la fecha, abarcando las décadas de los cincuenta y sesenta del pasado siglo, etapa que podemos decir describe un ciclo económico completo al comprender una primera parte de prosperidad (1853-54 a 1864) y una segunda fase de claro declive a partir de 1864 . La primera nota que caracteriza las relaciones 
mantenidas con el exterior es la preeminencia del Reino Unido, que siempre significa del 30 al $40 \%$ del comercio realizado con otros países. Francia, en segundo lugar, experimenta un crecimiento en su participación en los momentos de mayor auge comercial, alcanzando hacia 1860 un cuarto del comercio total, para declinar después a las cifras de partida que le adjudicaban entre un 12 a un $14 \%$. Con Italia experimentamos un incremento en nuestras relaciones bilaterales digno de mención, pues de un 3,6\% al principio se llega al 15\% en 1872 , aunque un año más tarde se desciende al $9 \%$. Suerte inversa contemplan nuestras relaciones con los EE.UU. o con Rusia. El 10\% que se alcanza con los barcos del primer país en 1856 queda reducido a un $5 \%$, y los rusos, que nunca sobrepasan el $5 \%$ en su concurso, a penas son el $2 \%$ al final del período. Por su parte, los alemanes se muestran estables en torno al 5\%. Finalmente, las cifras que nos relacionan con Portugal deben estar sometidas a la consideración del comercio de cabotaje realizado con las localidades vecinas del sur de aquel país, que una vez son objeto de atención estadística contribuyen a ampliar hasta el $14 \%$ de 1872 y 1873 , el modesto $3 \%$ y $7 \%$ de fechas anteriores. En todo caso, no se puede olvidar que la suma conjunta del comercio realizado bajo bandera británica o francesa supera en todo tiempo la mitad del comercio con el exterior (4).

\section{LAS MERCANCIAS}

Los buques que entran y salen del puerto declaran la carga que transportan en 2.676 y 1.238 ocasiones, respectivamente. De estas cifras globales tendríamos que descontar el transporte que se efectúa bajo las denominaciones de "carga general" o "varios efectos", puesto que resulta de todo punto imposible averiguar la naturaleza última de las mercancías que comprenden y que llegan a ser el 18\% del total transportado. Además, en el apartado de las salidas habría que considerar los buques que atracan en condición de "tránsito", el 6,5\% del total, expresión que nos indica que estamos ante embarcaciones que tan sólo han utilizado Cádiz como puerto de escala, sin que haya habido ningún trasvase de mercancías, lo cual resta importancia al desconocimiento de los productos que llevan en sus bodegas.

(4) Las cifras han sido tomadas del Boletin Oficial de la Provincia de Cádiz, que refleja el movimiento portuario en cada año, en este caso se ha tenido en cuenta la relación que va de 1856 a 1873 . 
Es relevante el número de barcos que transitan de vacío, lastrada su zona de carga con arena o agua para no perder estabilidad en la navegación. Lo significativo de su concurso, tanto si adoptamos el punto de vista de las llegadas como el de las salidas, nos ha conducido a contemplar estos casos que, en puridad, hacen referencia a una ausencia total de carga. Ocupan el primer lugar, con 395 barcos, entre los llegados, casi un 15\% del conjunto, aunque reducen su participación en las salidas, con sólo un 6,6\% del total que forman las 82 embarcaciones que se van de vacío. Destacable, por tanto, resulta ser la presencia expresa de un gran número de embarcaciones que acuden a Cádiz sin que la ciudad requiera nada de ellas, con el único propósito de obtener mercancías específicas de la región.

Dentro del variado espectro de bienes que se nos ofrecen como objeto de comercio, una primera puntualización que discrimine entre productos comprados y vendidos se hace necesaria. Se aprecia perfectamente como es más diverso el número de objetos demandados por la región que los ofertados por la misma. La tipología de las entradas afecta a 100 mercancías diferentes, mientras que los bienes de consumo exterior apenas alcanzan la mitad de esta cantidad. Además, si bien dentro de la carga entrada no existe ningún producto dominante que supere el 10\% del total de llegadas (el carbón roza ese porcentaje con un $9,9 \%$ y el vino se sitúa inmediatamente por detrás con un $7,6 \%$, no siendo desdeñable el concurso de la pesca que significa otro $10 \%$ del conjunto si sumamos las entradas de "pescado y "sardinas"), en las salidas sí podemos hablar de dominio indiscutible de dos productos esenciales para la exportación, la sal que, de forma destacada, acapara nada menos que el $35,5 \%$ de las salidas, y el vino con un, más modesto, $14,2 \%$.

Bien es cierto que, en virtud de la advertencia hecha acerca de la diferencia numérica entre el total de entradas y salidas, es posible pensar que el amplio abanico que compone el consumo interno está engrosado por el tráfico de cabotaje realizado desde los puertos más cercanos a la capital gaditana, que no queda registrado, en cambio, en el caso de las salidas. De todas formas, la carencia de información al respecto no parece casual y más bien nos atrevemos a sospechar que esos faluchos, laúdes o místicos vuelven a sus puertos de origen de vacío, de ahí que no se haya considerado necesario su recuento en el regreso, y, de ser así, su exclusión no alteraría los datos obtenidos para el conjunto de las exportaciones.

Entrando en la relación de productos consignados (ver lista completa en los anexos), resultan claramente dominantes los produc- 
tos agrarios, pecuarios y pesqueros. Estos son el $40 \%$, redondeando cifras, de las entradas y alcanzan el 58,6\% de las salidas: vino, frutos, naranjas, higos, cebada, trigo y papas, principalmente, por el primer capítulo; huevos, cerdos y chacinas en general, por el segundo; finalmente, bajo la denominación genérica de pescado y específica de sardinas, bacalao, atún y mojama, obtenemos la relación de productos de la actividad pesquera. De las mercancías que restan resulta descollante el concurso del carbón, con 266 buques llegados y tan sólo 5 salidos, amén de los ladrillos, las duelas, la madera, el esparto, el petróleo, la loza o las losas, el jabón, el cuero, el hierro y su variante en tubos o traviesas, el tabaco, todo ello entre las entradas; y las duelas, el cuero, el plomo y otros diferentes minerales, el corcho, el tabaco, para las salidas, que componen el $26 \%$ de los productos demandados y son, a su vez, el $8 \%$ de los ofertados desde el muelle gaditano.

Es claro que un recuento de lo cargado nos acercará al tipo de actividad económica desarrollada por el puerto, y así el muelle gaditano se nos ha descubierto como un punto de recepción de las principales mercancias que van a ser posteriormente distribuidas en el entorno peninsular. Además, a esta tradicional actuación de intermediario se suma su vocación exportadora en dos productos básicos, la sal y el vino. Es de justicia reconocer la supremacía de la sal marina en cuanto al número de navíos que se interesan por este tipo de carga, por encima de la indiscutible atracción que suponen los caldos de la tierra: recordemos que son un $35 \%$ de las unidades totales frente a un $14 \%$ que alcanza el vino. Sin embargo, y a pesar del claro predominio de la actividad salinera proyectada hacia el exterior, estaría por ver cuál es el producto extraído finalmente de este movimiento, cuál es el valor económico de los negocios que mueven la sal y el vino comparativamente. Es aquí donde parece trastocarse la fácil deducción que estaríamos tentados a establecer entre un mayor volumen de transacciones y un mayor rendimiento de las mismas. Si consideramos fiables las estimaciones del cónsul de Francia emitidas en sus informes administrativos relativos a la actividad exportadora desarrollada por el puerto hacia 1873, la cantidad en francos rondaría los 77,8 millones, de los que al menos 73,4 corresponderían al negocio vinatero que no hace sino crecer en esos años hasta llegar a la cifra indicada que establece el record del siglo (exactamente se habría superado en 11 millones los beneficios del año anterior), mientras que el espectacular trasvase salinero que se despliega apenas sí sobrepasaría el millón de francos anuales. 
Para completar los datos relativos al comercio globalmente considerado y sabiendo que son 93,7 millones de francos el valor del movimiento portuario conseguido ese año, es fácil deducir que casi 16 millones corresponderían a las importaciones, una vez restados los 77,8 millones exportados. Por lo tanto, se acentúa la tendencia señalada en su día por el profesor Nicolás Sánchez-Albornoz, que apreciaba a partir de la crisis de los sesenta una caída en picado del consumo interno representada por la reducción casi a la mitad de las importaciones, al mismo tiempo que los valores totales se estabilizan en torno a los 80 y 90 millones, gracias al crecimiento paralelo del valor de lo exportado, cuya rentabilidad última, si tomamos en consideración las apreciaciones consulares, se debe al incremento espectacular de la salida de los vinos de la zona. El cuadro que seguidamente introducimos ratifica fielmente el comentario realizado, las importaciones habían subido a partir de esa fecha un 11\%, el negocio global, sin embargo, era menor en un $23,3 \%$.

Aunque no entra de lleno en la investigación que aquí estamos realizando, no hemos resistido el avanzar la posible tendencia seguida por los negocios camino del fin de siglo. De esta manera, y también extraído de los informes consulares citados, incluimos el valor de los intercambios correspondientes a 1880 y 1881 donde apreciamos, por un lado, un mantenimiento de los niveles de ingresos desde las últimas fechas, 87,8 y 96,6 millones de francos, respectivamente, y por otro, un reparto porcentual más equitativo entre importaciones y exportaciones, donde las primeras recuperan posiciones situándose en torno al $40 \%$ del total de las transacciones, mientras las segundas pierden peso y se estabilizan en un $60 \%$ de las mismas. También son de Benedetti las cifras que en el cuadro 3 reflejan el beneficio obtenido por los extractores de sal, sorprediéndonos el escaso resultado en relación al movimiento salinero que se da en el puerto y que muestra cómo el año republicano no es precisamente el mejor de toda la serie, con un $36 \%$ menos de lo conseguido el año anterior. Es muy probable que el negocio salinero se viera favorecido por la supresión del monopolio que el Estado tenía sobre la venta del producto, bien acogida por la opinión gaditana, proclive también al desestanco del tabaco (5).

(5) C.C.C., despacho № 256, de 13 de enero de 1870, pp. 222-227. 


\section{RUTAS Y PUERTOS DE COMERCIO}

Es tiempo ya de que especifiquemos cuáles son las áreas geográficas que abarca este flujo mercantil. El gráfico que aquí introducimos, confeccionado a partir de los puertos de procedencia y destino declarados por cada embarcación, se ocupa de descubrir las zonas elegidas por el comercio que se hace desde o a través de nuestra ciudad (al final del trabajo se ofrece una lista completa de la relación de puertos y el número de entradas y salidas que a cada uno corresponde).

Gráfica 6: Distribución continental del comercio gaditano

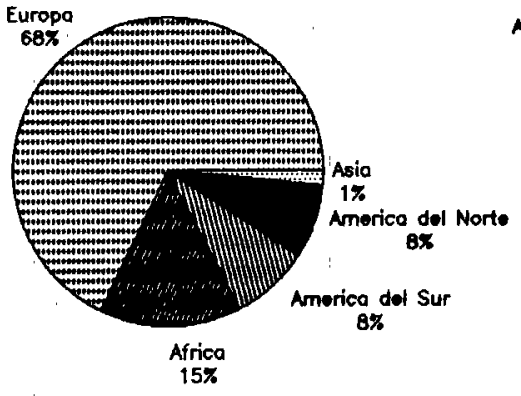

ENTRADAS

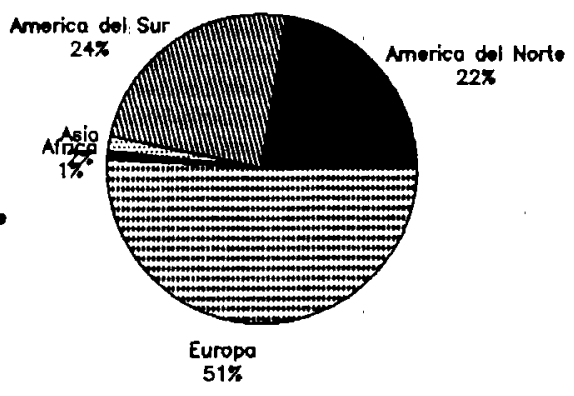

SALIDAS

La primera consideración que se desprende de la simple observación de ambas tartas representativas, en las que han sido intencionadamente excluidos los puertos españoles que serán objeto de atención más adelante, es que el comercio con Europa domina, con diferencia (es el. 68\% de las entradas y algo más de la mitad, el 51\% de las salidas), las relaciones internacionales que se mantienen. Hemos preferido distinguir en las rutas que se dirigen o vienen de América el transporte que implica al Norte o al Sur de aquel continente, con el fin de calibrar el estado en que se encuentran los tradicionales lazos comerciales con las viejas posesiones de ultramar, ahora reducidas al espacio isleño del Caribe. Es claro que, siendo muy modesto el concurso de las entradas americanas, Norteamérica y Sudamérica se reparten con equidad el $16 \%$ del comercio total, mientras que los viajes emprendidos hacia estos territorios del otro lado del Atlántico concentran el $46 \%$ de los envíos, repartidos igualmente a medias. Tras las cifras concernientes a Europa y América, que están cerca de ser el cien por cien del comercio global, queda bastante mermada la importancia del tráfico africano o asiático que se realiza a través de nuestro puerto. Obviamente, el vínculo que obligadamente mantiene España con las Filipinas, en el 
desempeño de su papel de metrópoli, concede ese pequeño 1\% a los contactos mantenidos con Asia, descontados dos viajes de entrada, uno desde Bombay y otro desde Singapur. Frutos, tabaco y varios efectos se traen desde Manila, de regreso, siempre haciendo escala en Barcelona y Singapur, domina la carga general. En cuanto a la participación del continente africano en el circuito comercial, el 15\% que le corresponde en concepto de envíos se refiere, hecha la salvedad de los 10 viajes que las fragatas francesas protagonizan desde las islas de Reunión y Mauricio con el azúcar que va a ser desembarcado finalmente en Nantes o en Marsella, al comercio de cabotaje que parte de los puertos norteafricanos de Tánger, Tetuán y Larache, con 57, 14 y 12 viajes, respectivamente, más otros desde Mazagán, Mogador, etc., con productos de directo consumo para los gaditanos que se transportan en faluchos y pequeños laúdes: huevos y gallinas de Tánger, peras y naranjas de Tetuán y Larache.

Siendo más importante el comercio realizado con América y Europa, parece oportuno, seguidamente, pormenorizar sobre las zonas de preferente interés. Por lo que respecta al espacio norteamericano habría que destacar dos áreas especialmente. De las inmediaciones de Terranova proviene la mayoría de los barcos, 9 en total, que traen el bacalao que se pesca por aquellos caladeros de altura. Son, sin embargo, mucho más importantes los viajes de regreso que realizan los 155 navíos cargados del material preciso para la elaboración de salazones. Al menos 113 de estas entregas se hacen bajo pabellón inglés, que controla de forma destacada este específico comercio, seguido, a bastante distancia, por los 24 franceses registrados. En los EE.UU., Nueva York con 37 salidas y 25 llegadas y Boston con 5 y 17, respectivamente, son los puertos más visitados. Las mercancías importadas son fundamentalmente el petróleo y las duelas, también el tabaco y un barco con algodón completan la lista de productos enviados. De nuevo, la sal y, ahora también, el vino constituyen los productos requeridos preferentemente por los estadounidenses. Si pasamos ahora al espacio de tradicional dominio español, el primer comentario obliga a dejar constancia de que las colonias que aún se mantienen, las islas de Cuba y Puerto Rico, significan las tres cuartas partes de las entradas sudamericanas. El azúcar, el tabaco, el café y, en general, lo estibado bajo la denominación de "frutos" proviene del espacio colonial. Lo poco que nos llega fuera de esta área queda reducido a algo de café y, sobre todo, a cuero argentino. El reflujo que nos relaciona con nuestras posesiones antillanas no llega a ser ni un tercio de los viajes de regreso con destino al cono sur americano. Los frutos y la sal son llevados por los correos que 
Antonio López tiene en concesión con el Gobierno desde 1861. Como algo característico de aquellos años, marcados por el conflicto bélico sostenido con los rebeldes de la Grande Antilla, es normal el desembarco de tropa en Puerto Rico. Fuera del área insular, fragatas, bergantines y goletas de diferentes países (italianas muchas veces, inglesas, noruegas, holandesas, francesas, alemanas, suecas, danesas y, también españolas), se dirigen hacia el ámbito rioplatense con la sal necesaria para el tasajo de la Pampa interior, con lo que se completa al menos otro tercio de los envíos a Sudamérica.

Dentro del contexto europeo, los muelles británicos absorben buena parte de la actividad portuaria vinculada a Cádiz. Por lo que se refiere al estado de los mercantes procedentes de dicho país, habría que indicar que optan entre llevar carbón en sus bodegas o entrar lastrados con el único propósito de cargar vino y/o sal en nuestra ciudad. El carbón sale de los puertos cercanos a las zonas mineras de aquel país, de Escocia, vía Glasgow, de donde salen además 8 buques que transportan tuberías, o de sus inmediaciones en Newcastle, Sunderland o Barrow, al norte de Inglaterra y de los yacimientos del País de Gales que tienen su punto de embarque en varias ciudades entre Liverpool, Cardiff (ésta última acapara el 35\% de los viajes) y Swansea, como centros principales. El sur de Inglaterra, desde Dover hasta Scilly, en el extremo occidental, pasando por Londres, componen con claridad las estibas en lastre británicas. Por lo que respecta a los viajes de regreso, el equilibrio relativo del que participaban los diferentes puertos británicos, se rompe en favor de la City londinense, que controla el 50\% de lo importado, y en esta ocasión es el vino lo que se desembarca primordialmente. Siguen en importancia, Liverpool y Glasgow que junto a Londres reciben las tres cuartas partes del comercio de exportación vinícola realizado con Gran Bretaña. Obviamente, el comercio con Irlanda resulta ser un apéndice del practicado con el área inglesa, con las mismas mercancías ya señaladas.

En Francia, Marsella es el lugar de partida para buena parte de los barcos que proceden de aquel país, pero, sobre todo, como le ocurre a Londres, se comporta como centro receptor contabilizando el $74 \%$ del total francés. El comercio con las ciudades atlánticas, desde Burdeos hasta Dunkerque, se reparte casi el $60 \%$ de los envíos hechos por los franceses, pero, al mismo tiempo, reducen ostensiblemente las importaciones, a excepción de El Havre, foco principal de la zona. Se puede decir, en líneas generales, que nada recibimos de nuestros vecinos galos, pues sus barcos nos visitan lastrados en la mayoría de los casos. Hay que hacer la salvedad de aquellos que proceden de los mares del 
sudeste africano cargados con el azúcar de Zanzíbar o Isla Mauricio, que tras hacer escala en nuestro puerto, también en algunos de Levante (punto obligado es Barcelona) se dirigen en su mayoría hacia Marsella y de ahí probablemente la carga pasa al tren de París. Ya destacamos en su momento la treintena de barcos que se dirigen con sal a Terranova (S. Pierre et Miquelon) y algún otro hacia Montevideo.

Los italianos, como ya fue apuntado, tienen en Génova el primer puerto de enlace con las aguas gaditanas. Sin embargo, estos barcos que, al igual que los franceses, acuden en lastre a nuestras costas, se limitan a cargar la sal que llevarán a los puertos sudamericanos ya conocidos de Montevideo, Río de la Plata, Bahía, Río Grande, y muy pocos volverán a hacer escala en Cádiz de regreso a Italia.

Poniendo orden en el espacio nórdico desde Bélgica hasta Rusia, los puntos de embarque o desembarque más usuales son el puerto belga de Amberes, la holandesa Amsterdam, Hamburgo en Alemania, Copenhague en Dinamarca, Christiansund o Bergen en Noruega, la Sueca Helsinfor y Lovisa o San Petersburgo en Finlandia y Rusia, respectivamente. Belgas y alemanes lastran sus barcos para venir de vacío o traen carga "general" o "espíritu" en el caso de algún alemán, los demás países transportan madera de los bosques del norte de Europa. Los noruegos se especializan, lo mismo que los ingleses que vienen de Terranova, en el bacalao. Todos, sin excepción, vuelven a los puntos de embarque con vino y/o sal.

Dentro ya de la Península Ibérica, el pescado que nos llega de Olhao y Fuseta hace de estos puertecitos del sur de Portugal los primeros en concurrir con nuestro comercio desde el país vecino. Si tenemos en cuenta los viajes de regreso, es la capital lisboeta la que recibe mayor número de barcos, exactamente 82 , procedentes de nuestra ciudad. Hay que puntualizar que Lisboa se comporta como punto de obligada escala camino de Londres, a la que se llega habiendo recalado previamente en el puerto gallego de Vigo. Nos imaginamos que el objeto de esta parada se justifica debidamente por el reclamo del Oporto que tanta aceptación tiene también en el consumo inglés y que sería allí estibado junto a nuestros caldos.

Pone punto final a este capítulo, la relación de puertos españoles que comercian con la ciudad gaditana. Se nos ofrece la oportunidad de profundizar en el propio circuito nacional de comercio de cabotaje, eclipsado en muchas ocasiones por el interés que despierta el intercambio internacional, pero no de menos valor que este último. El tráfico entre puntos de la costa española presenta un dominio casi absoluto del pabellón nacional, como es lógico suponer, y el primer punto de 
interés que descubrimos es que, grosso modo, existe un equilibrio relativo entre los viajes que se emprenden hacia las localidades del Mediterráneo o rumbo a los puertos bañados por el Atlántico (8).

Comenzando el comentario por la costa catalana, no resulta novedoso avanzar que Barcelona acapara la atención de la mayoría de los barcos que transitan por la zona, tanto en los viajes de ida como en los de vuelta, y supone un 4,6\% del tráfico nacional total. Las expresiones más utilizadas para describir el tráfico con la Ciudad Condal son las de "carga general", "varios efectos", "lastre", etc., aunque también son importantes las partidas de vino que de alli nos llegan. Barcelona recibe todas las mercancías que hemos visto desfilar por el puerto andaluz, donde barcos nacionales y extranjeros han hecho escala con duelas, petróleo, bacalao, café, carbón, frutos, azúcar, etc., por lo que es lógico pensar que Cádiz no es la meta final para estos navíos, sino tan sólo una escala intermedia hasta alcanzar el que sin duda ya es el primer puerto comercial de España (9). Vino y aguardiente nos traen también los barcos llegados del entorno catalán (Tarragona, Vendrell, San Carlos de la Rápita y Vinaroz), el papel y la cebada son otras mercancías con la misma procedencia. De los puertos mallorquines (Andraitx, Ibiza...) nos llega jabón y, en contrapartida, Palma, la capital, como Barcelona, recibe productos muy variados: trigo, café, cueros, aceite, azúcar, etc.

Pasando a la cosa levantina, entre Castellón y Almería se suman al jabón y a la cebada, el arroz valenciano, la sal de Santa Pola y Torrevieja y el esparto surestino que nos llega desde Alicante, Aguilas y sobre todo desde Almería. Papas, batatas, higos y habichuelas provienen de los pueblos costeros situados entre esta última ciudad y la malagueña Estepona. Además, algún mineral como el plomo embarcado en Adra y el hierro de las ferrerías del interior que se estiba en Velez-Málaga y Fuengirola, constituyen los envíos de la Andalucía oriental. Del puerto malacitano un sinfín de productos, como corres-

(8). En las entradas se realizan 834 viajes rumbo al Este y 953 hacia el Oeste; en las salidas, 407 y 346, respectivamente. Volvemos a recordar que la diferencia entre el número de entradas y salidas con carga declarada desvela el concurso del tránsito de barcos menores desde un radio próximo a la ciudad para el caso de las entradas que no son consideradas en los viajes de regreso.

(9) El desplazamiento del puerto de Cádiz a un segundo lugar en importancia tiene una fecha bastante reciente según testimonio de Nicolás Sánchez-Albornoz, al mostrar que el volumen total de negocios de 1849 todavia es favorable a la ciudad andaluza con 82,2 contra 76,5 millones de francos, relación que ya se ha invertido en 1857, fecha en la que el saldo gaditano, con ser creciente, es de 93 millones, a distancia ya de los espectaculares 174 millones de la Ciudad Condal. Nicolás Sánchez-Albornoz, Op. Cit., p. 89. 
ponde a la segunda ciudad en importancia con la que entra en contacto el puerto gaditano, ya que un $14,5 \%$ de las entradas y salidas totales van y vienen desde allí. Destaca, sin embargo, el vino, la cebada y la loza entre los principales productos malagueños. Málaga recibe, como Barcelona, las mismas mercancías que han entrado en el puerto gaditano desde el exterior, ocupando un lugar preferente las duelas que se van emplear en el envejecimiento del vino local. Importantes son los correos regulares que unen por barco a Cádiz con las poblaciones de Tarifa y Algeciras, para terminar en la británica Gibraltar. Es imaginable lo común que llega a ser el tráfico inglés por la zona del Estrecho con base en el puerto gibraltareño, del que los gaditanos envidiaban, por aquellas fechas, su especial status de franquicia que lo convertía en un fuerte competidor (10). Algeciras participa de casi el 10\% del comercio total: el vino, la cebada, el jabón y el esparto, aparecen en la lista de productos llegados desde allí, y a esto se añaden especialmente el carbón y las losas enviadas también desde Tarifa.

Mirando ya hacia el Atlántico, las poblaciones costeras de Huelva se especializan en diferentes mercancías. Si hablamos de Bonanza, Moguer o Cartaya, sería preciso referimos a los aportes del vino que, una vez en nuestra ciudad, se intentará hacer pasar por el mejor caldo jerezano con miras a la exportación (11). El pescado y las sardinas provienen de Isla Cristina y Ayamonte. Con el otro espacio insular, Las Canarias, el Gobierno español también ha concertado varios vaporescorreos regulares que tienen su punto de habitual embarque en Cádiz con destino a Tenerife.

(10) "... a algunas millas de distancia prospera y florece una colonia de la Gran Bretaña; colonia fundada en un seco y árido peñón, que mientras fue de España nada valió, nada produjo y que hoy viene a ser con respecto a Cádiz lo que esas plantas parásitas que extendiendo por debajo de la tierra sus largos filamentos...", así describía la situación de Gibraltar un publicista del Diario de Cádiz, № 1. 252, de 19 de febrero de 1873.

(11) La depreciación del vino jerezano en las décadas de los sesenta y setenta del pasado siglo fue contrarrestada por los extractores, con objeto de mantener el nivel de beneficios, con un aumento de la producción y un incremento de las exportaciones muy considerable. Para atender al creciente volumen exportado, se recurrió a tierras de peor calidad, pero también a zonas que quedaban fuera de lo que entendemos hoy pertenecerían a la comarca natural de crianza de estos caldos. Los barcos de Huelva que atracan en Cádiz con vino responden a esta demanda suplementaria que muchos, entre los que cabe destacar al cónsul de los EE.UU. en la ciudad, Mister Farrell, consideraban sin ambages como fraudulenta. Hemos tenido ocasión de profundizar sobre el tema en "exportación y fraude en el comercio del vino: los informes del cónsul norteamericano en Cádiz (1866-1867)", en Revista de Historia de Jerez, № 1, 1992, pp. 29-41. 
Antes de ascender por el Atlántico y alcanzar los puertos del Norte, no podemos dejarnos atrás el puerto que, por el número de barcos que de allí nos vienen y que allí van, ocupa el primer lugar entre todos; nos estamos refiriendo al estuario sevillano sobre el Guadalquivir, que funciona como un apéndice fluvial para las mercancías que desde todos los puntos que hemos visto llegan a Cádiz, con la intención última de penetrar hacia la zona del interior. Sevilla está próxima al $20 \%$ del intercambio globalmente considerado. Fundamentalmente, son productos agrarios los que bajan por el río hasta nosotros: el trigo, las aceitunas y el aceite son los principales, siguen las papas y el maíz, y destacaríamos también los ladrillos y la loza sevillanas.

Vigo, Santander y Bilbao son los puntos elegidos preferentemente para embarque y desembarque en el Norte. El primero de ellos significa algo más del $8 \%$ de todo el comercio nacional y es escala obligada entre Lisboa y Londres. La Coruña y Gijón ocupan lugares secundarios. Maíz, madera, carbón, sardinas, cal y tubos son los envíos más frecuentes desde cualquier punto de la cornisa cantábrica, recibiendo a cambio nuestra sal que tan apreciada es en el extranjero.

\section{LOS BARCOS}

No cabe duda que uno de los aspectos más descollantes acaecidos a raíz de la Revolución Industrial ha sido el progresivo aumento de la capacidad inventiva del hombre puesta al servicio del desarrollo productivo sin precedentes que ésta provoca. El siglo XIX, por tanto, se encuentra plagado de hitos en la investigación tecnológica, que han dado como resultado con su aplicación un salto adelante dentro de la actividad económica mundial. La marina mercante y la navegación, en general, no escapa al influjo investigador de la época, sino que, muy al contrario, es uno de los principales sectores que se benefician de esta ampliación del conocimiento científico que corresponde a la denominada "Segunda Revolución Industrial", de manera que, junto a la innovación en las comunicaciones terrestres que representa el ferrocarril, la modernización de la flota existente revoluciona el transporte de mercancías por mar, medio, no hay que olvidar, que domina en la pasada centuria el flujo mundial de mercancías.

Desde que Fulton mostrara a Napoleón, hacia 1803, las posibilidades de aplicación de la fuerza motriz del vapor en el tránsito fluvial 
del Sena (12), la transformación de la marina a vela podía darse como un hecho consumado. No obstante, los lentos vapores de ruedas de la primera mitad del XIX a duras penas podían competir con los rápidos veleros de la época, que llegan a su máxima perfección justo entonces. El mito de los fusiformes y marineros clipers norteamericanos, que alcanzan su merecida fama tras la Guerra de Secesión americana y que concursan sin posible competidor en la carrera del opio, es parejo al perfeccionamiento de los buques movidos por calderas que, en principio, no pueden renunciar a llevar aparejo blanco porque resulta dificil abastecerse de carbón en las largas rutas que emprenden. Con la hélice, que mediada la centuria comienza a sustituir a la pesada rueda central del casco, se inicia la mayoría de edad para los barcos de vapor, que consiguen una velocidad punta que los hacen competitivos ante el hermano mayor movido por el viento. Paralelamente, la sustitución de la madera por el hierro en los cascos de todas estas embarcaciones contribuye al incremento del tonelaje mundial con unidades cada vez más grandes y más pesadas que proporcionan mayores posibilidades para la carga, reduciendo los fletes de manera considerable. A fines del XIX se experimentará con el motor de petróleo, con lo que se abren nuevas expectativas también para la navegación, sólo que el alcance de este invento traspasa la frontera del siglo y por consiguiente queda fuera del marco cronológico de interés que nos hemos trazado.

El primer servicio de vapor a ruedas español cubría el trayecto marítimo y fluvial entre Cádiz y Sevilla, con escala en Sanlúcar (13). De entonces para acá, el vapor se fue introduciendo paulatinamente entre nuestra flota, a un ritmo que iba a la zaga del contexto mundial. Si en 1846 las estimaciones del tonelaje en todo el mundo daban una proporción de un escaso $10 \%$ para esta fuerza motriz, en 1875 de los 20 millones de toneladas mundiales, un $25 \%$ quedaba reservado a ésta (14). Una fecha como 1868 sirve para evaluar la situación española, que arroja un $12 \%$ de unidades a vapor y el mismo porcentaje en el tonelaje total nacional. Ya en vísperas de la primera conflagración mundial, la marina mercante de todo el globo se movia en más de un $90 \%$ con la ayuda del vapor o motor de petróleo, y su tonelaje, igualmente,

(12) Maurice de BROSSARD, Historia Marítima del Mundo. Ediciones Amaika, Barcelona, 1976, vol. 2, p. 208.

(13) José CERVERA PERY, La Marina Mercante Española. Historia y circunstancia. Editorial San Martín, Madrid, 1990, p. 68.

(14) Maurice de BROSSARD, Op. Cit., p. 219. 
acaparaba casi el ciento por ciento de lo transportado, la vela había quedado para el recreo o para los nostálgicos de viejas aventuras de piratas (15).

Pero, volviendo a las fechas que aquí nos interesan y considerando el cómputo de los valores como un índice que revela el grado de modernización de la flota mercante que entra y sale del puerto gaditano, en el año republicano son 968 las embarcaciones de ese tipo que tocan nuestro puerto, lo que supone alrededor de un 31\% del conjunto, el resto se mueve aún bajo el régimen que establecen los vientos. La evolución del número de vapores tiene que ir forzosamente en aumento como signo inequívoco de adecuación a las mejoras técnicas introducidas en el transporte marítimo, y así en 1880 y en 1884 son ya 1.303 y 1.426 , respectivamente, los buques movidos a vapor y su porcentaje va creciendo poco a poco del mismo modo, un $35 \%$ para el primer caso y un 37\% para el segundo; comenzando el siglo XX, alrededor del $60 \%$ de los barcos que nos visitan son de esta clase (16). Posiblemente podemos aventurar que el espectacular aumento del tonelaje movido que se aprecia en las gráficas 2 y 3 hacia finales de siglo, en contraposición a la merma considerable del número de buques que contabiliza el gráfico 1, se debe al concurso mayor, y más pesado, de estos vapores construidos en hierro que desplazan definitivamente a los cascos de madera movidos tradicionalmente a vela. Estos últimos componen el 69\% que falta para obtener el cien por cien de los barcos que entran en 1873 , aunque hay que advertir que, descontados los tipos menores, las goletas, polacras, fragatas y bergantines que componen los buques mayores a vela son algo menos de la mitad del tonelaje desplazado por estos grandes mercantes, de tal manera que un $57,7 \%$ de las entradas (el 51\% de las salidas registradas) de este transporte de tipo mayor se hace ya en vapores.

(15) Evolución del número de barcos y tonelaje correspondiente a la flota nacional, extraído de la obra de José Cervera Pery, Op. Cit. pp. 77 a 117.

\begin{tabular}{rrrrrrrrr}
\multicolumn{2}{c}{ Veleros } & \% & Toneladas & $\mathbf{\%}$ & Vapores & $\mathbf{\%}$ & Toneladas & $\%$ \\
1868 & 1.312 & 88 & 147.160 & 88 & 180 & 12 & 20.814 & 12 \\
1911 & 301 & 34 & 44.325 & 6 & 582 & 66 & 750.081 & 94 \\
1920 & 568 & 49 & 99.369 & 11 & 582 & 51 & 826.905 & 89 \\
1934 & 119 & 13 & 20.296 & 2 & 833 & 87 & 1.155 .806 & 98
\end{tabular}

(16) Antonio POLEY Y POLEY, Cádiz y su provincia. Descripción geográfica y estadística ilustrada con mapas. Tipografía de E. López y Cáa, Sevilla, 1901, p. 158. 
Pasando al ámbito de la vela, de sobra es conocido que las características del aparejo utilizado por cada barco da nombre genérico al tipo de embarcación al que pertenece. Las dimensiones de estos navíos, como nos recuerda el profesor García-Baquero, pueden también relacionarse con la tipología establecida, aunque, bien es verdad, que resulta difícil mostrar diferencias entre ellos en razón única de su desplazamiento (17). Es, pues, el estudio del velamen empleado el factor que normalmente nos define la embarcación en cada caso, aunque es cierto que podemos hacernos una idea aproximada en algunos casos del tamaño del buque según el tipo que definen las velas que emplean. Grosso modo podemos entender, volviendo a la relación con la que trabajamos, como embarcaciones mayores de vela a las barcas, bricbarcas, fragatas, bergantines, polacras, goletas, pailebotes y queches que entran y salen de nuestro puerto, dando la categoría de menores a los laúdes, místicos, faluchos, pataches, charangueros y botes, más las lusitanas canoas y los caiques también típicos del vecino país; finalmente la balandra o balandrita estaría a medio camino entre el grupo de los grandes y los pequeños veleros. En cuanto al aparejo propio de todos ellos y resumiendo los aspectos más esenciales, hay que recordar que se llama fragata a toda embarcación de tres palos (trinquete, mayor y mesana) con velas cuadras, es decir, perpendiculares a la línea del casco; bergantines cuando sólo hay dos palos con las mismas características, y goletas cuando son dos palos aparejados a cuchillo, queremos decir en línea con el casco. Las variantes que pueden establecerse a partir de estos tipos son varias: tendremos una bric-barca si aparejamos la última vela de la fragata, la del palo de mesana, con cangreja (vela trapezoidal); estaremos ante un bergantín-goleta, muy numerosos en Cádiz por aquellas fechas puesto que son algo más de la mitad de los bergantines anclados, si el primer palo va con vela cuadra, y el segundo, o restantes, si los hay, con velas a cuchillo; por último, denominaremos polacra a una especie de bergantín que no lleva masteleros ni cofa, que puede formar un híbrido con la goleta si tiene el palo mayor como ésta y que se denomina polacra-goleta, claramente mayoritaria en el registro de polacras gaditanas con 43 unidades de las 50 contabilizadas.

(17) Antonio GARCIA-BAQUERO en Cádiz y el Atlântico (1717-1778). Publicaciones de la Escuela de Estudios Hispano-Americanos de Sevilla, 1976, vol. I pp. 239240 , intenta determinar el tonelaje de los veleros de la carrera de Indias, aunque termina por considerar oscilaciones importantes dentro de cada tipo. Por ejemplo, la fragata podría ir de 52 ó 76 toneladas a 498, la balandra desde 40 a 176, la goleta de 45 a 143 y la polacra, de 75 a 173 . 
La hermana menor de estos veleros, la balandra, tan sólo lleva un palo aparejado, el mayor; a continuación, los laúdes y faluchos son pequeñas embarcaciones de casco largo y estrecho que soportan vela latina y son propias del Mediterráneo. El místico, el grande entre los pequeños, puede llevar de dos a tres palos con aparejo semejante al latino, y también es común al ámbito Mediterráneo. Hemos indicado que el caique es la típica embarcación de pesca portuguesa, aunque no es extraña al litoral levantino; además está la canoa, que trae el pescado hasta nuestra costa. Por fin, el charanguero, barco costero andaluz utilizado en el tránsito por el Guadalquivir, lleva aparejo latino y no tiene cubierta.

Gráfica 7: Tipología de los barcos arribados a Cádiz
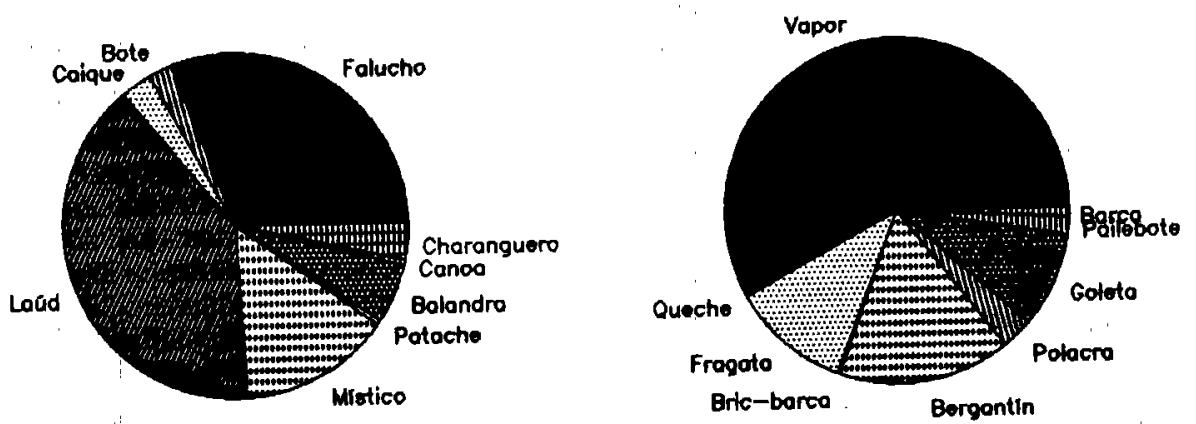

Junto a esta descripción técnica de las características esenciales de los buques, merece la pena descubrir qué funciones son comunes en cada tipo. Efectivamente, la bric-barca y la fragata debían constituir los grandes entre los veleros, puesto que lo normal es que realicen los viajes de mayor distancia, desde o hacia el Báltico (Lovisa, Helsinfors, Carlsham etc..), o desde o hacia el otro lado del Atlántico (especialmente tocan puertos norteamericanos). El bergatín juega un poco el papel de comodín entre los de su familia a vela. Debía haber diferencias notables en el tamaño de los de su clase, lo que parece corroborar su clasificación en puros bergantines (122 unidades) y bergantinesgoletas (151 unidades). Al bergantín no se le resiste ni la larga ni la corta distancia, es capaz de hacer rutas de cabotaje o cruzar el océano sin problemas; no es extraño, por tanto, que sea el más numeroso entre los veleros, por delante de las fragatas. El papel de las goletas está restringido a 151 embarcaciones, cantidad inferior a la de los tipos anteriores. Se especializa en el cabotaje y es particularmente utilizada por el pabellón nacional entre puertos españoles, pero, asimismo, las goletas están capacitadas para alcanzar distancias mayores que las 
relacionan con muelles británicos primordialmente (Newcastle, Cardiff, Dartmouth, Plymouth, etc.), y si consideramos las salidas de las inglesas, observamos cómo emprenden valientemente el viaje hacia las inquietantes aguas del norte, enlazando con Terranova, Gaspe o, más al sur, hacia Río Grande, para llevar en sus bodegas la sal requerida en estos destinos. Goleta era el "Estamos Aquí" de la famosa novela de R. Kipling Capitanes intrépidos, que pretendía estar sin tocar tierra desde mayo hasta septiembre, el tiempo que duraba la temporada de pesca del bacalao en los caladeros de Terranova, para lo cual llevaba en sus bodegas, quién sabe si estibados en Cádiz, 100 barriles de sal con la que conservar tanto pescado. La polacra (en su mayoría polacra-goleta en 43 de las 50 ocasiones y españolas en su registro, 48 del total) es usada también en la circunnavegación de España y no son pocas las que se aventuran hacia el mar Caribe, disponiendo la proa hacia la colonia antillana. Por último, la balandra o balandrita, la menor entre los grandes, con 78 unidades, todas españolas, recorre fundamentalmente la ruta mediterránea entre puertos nacionales y también es utilizada para ir y venir de Sevilla y de Huelva.

Si el caique y la canoa, como dijimos repetidas veces, son pequeñas embarcaciones portuguesas que traen pescado, un buen número de místicos ( 53 de los 198 que suman el total) provienen de aquel país con la misma carga desde su base en Olhao o Fuseta. El resto, de bandera española, hace trayectos de cercanías que no sebrepasan Algeciras por el Este, llegando a Huelva y a sus pueblos del litoral como Moguer, Cartaya o Bonanza, por Poniente.

El falucho y el laúd despliegan su vela latina en rutas parecidas, si bien el primero queda reservado para puertos próximos (todos los pueblos costeros onubenses, Sevilla y algunos del norte de Africa, como Tánger o Tetuán). El laúd, además de este mismo recorrido, cubre distancias mayores, siempre sin perder de vista la costa mediterránea: Adra, Nerja, Málaga, Almería, Albuñol, Vinaroz, Andraitx, Vendrell, Tarragona, etc. Por último, el bote, como es de suponer, no traspasa el radio de Sevilla, Huelva y Tánger.

Hemos dejado para el final los vapores, que son el 75\% de los grandes mercantes españoles y buena parte de la escuadra inglesa, francesa y alemana, con un 44\%, 36\% y 55\% de sus respectivas flotas. Es significativo que los países más alejados de nosotros, EE.UU., Rusia, Holanda, Noruega, Suecia o Dinamarca, utilicen en sus singladuras preferentemente veleros que se adaptan mejor a los largos recorridos. Los italianos, que en principio provienen desde la cercana Génova, constituyen un caso especial al hacer uso destacado de la fragata (el $62 \%$ de 
sus barcos) y del bergantín ( $24 \%$ de los casos), nada extraño, por otra parte, si caemos en la cuenta que vienen a cargar la sal que han de llevar al Nuevo Mundo. Así, pues, el vapor es el buque preferido por los nacionales para realizar la circunvalación de la Península Ibérica, también es el barco elegido por los ingleses que provienen del Peñón, para, desde aquí, emprender el regreso a su país. Es la embarcación utilizada por británicos y españoles para llevar el vino, previa escala en Lisboa y Vigo, hacia la capital londinense. Franceses y españoles lo adoptan para sus viajes a Marsella y, por fin, los alemanes van y vienen con el vapor desde Hamburgo.

\section{LOS CONSIGNATARIOS}

Reciben este nombre, según definición del Diccionario de la Real Academia de la Lengua, todo aquel que en los puertos de mar representa al armador de un buque para entender de los asuntos administrativos que se relacionan con su carga y pasaje, definición que viene a coincidir con la usual del Derecho Marítimo, que también le hace responsable de los trámites mercantiles; sin embargo, el Código de Comercio le adjudica otra acepción al presentarlo como la persona a la que va destinada la carga. Teniendo en cuenta la ambivalencia de su gestión comercial, nos interesamos ahora por los nombres que están detrás de todo este trasiego de bienes, aquellos que se benefician en primer término del comercio marítimo gaditano. Hemos querido recabar la mayor información posible de los mismos, no contentándonos exclusivamente con los datos que son fáciles de obtener a partir del registro de entrada que refleja $E l$ Comercio y que es básico para esta investigación; con él hemos podido precisar el volumen de negocios imputable a cada uno de ellos, del mismo modo que profundizando en ello hemos apreciado evidentes casos de especialización circunscrita a determinados ámbitos de comercio. Además de esto, hemos tratado de averiguar otros aspectos más personales procediendo a la localización de estos hombres de negocios en el Padrón de Vecinos de 1873 y, en su defecto, en los de 1870 y 1872, para obtener así referencia exacta del lugar de nacimiento, edad y estado civil de los mismos, así como otros aspectos que los terminan por ubicar dentro de la burguesía gaditana; nos referimos al lugar de residencia, a la contribución que satisfacen y al número de sirvientes que tienen a su cargo.

Los 41 nombres de la lista que al final se ofrecen fijan su lugar de residencia preferentemente en los barrios de San Francisco y San Carlos, Correo, Cortes, Constitución y Pópulo. Se trata de espacios, especial- 
mente los cuatro primeros, de clase acomodada, lo que se aprecia en la composición profesional dominante de sus residentes, como se ha podido comprobar en otros trabajos (18). Para poder ser más concluyente en este sentido, se ha evaluado a través de las contribuciones declaradas en una fuente pertinente, el nivel que alcanzan los negocios que, bajo la expresión profesional de "comercio" o "comerciante" se atribuyen (19). Gracias a esto sabemos que superan las 1.000 pts. fiscales 15 de ellos (13 tan sólo por subsidio industrial y de comercio) y otros 4 quedan muy cerca de esta cantidad con 950 pts. Se puede decir de ellos que pertenecen al nivel económico más alto de la ciudad, puesto que, dentro de esta categoría sólo contribuyen 210 familias de las que 85 lo hacen estrictamente por el capítulo de subsidio industrial. En una escala menor, pero siempre superando las 500 pts. de contribución que les asegura su permanencia entre los miembros de la clase media alta, están todos los restantes, salvo un caso de contribución más modesta correspondiente a Cristóbal Colón. Es una lástima que algunos de ellos no hayan cumplimentado la información económica requerida por los republicanos en 1873, privándonos de la oportunidad de calibrar su, probablemente, desahogado nivel de vida; sin embargo, dispuestos a solventar las deficiencias con que algunas fuentes históricas llegan hasta nosotros, buscamos alternativamente en otro indicador la solución a este problema. En este sentido, el número de sirvientes que asisten con su trabajo a cada una de las familias ha ayudado a ubicarlos sociológicamente. Lo normal es que se disponga de más de un sirviente, entre dos y cinco domésticos empleados tienen 18 nombres de la lista, y superan el número de 6 cuatro de ellos. No es raro, para finalizar brevemente estas consideraciones de tipo personal y familiar, que los negocios se lleven entre hermanos, tal es el caso de los Alcón, Mac-Pherson, Retortillo y Rudolph, o entre padre e hijos, como los Martínez de Pinillos, Sicre o Sobrino. Otra característica fácil de descubrir es la presencia destacada de extranjeros al frente de los negocios propios de sus países de origen, a los que representan diplomáticamente en buena parte de los casos.

(18) Me remito al trabajo de Alberto Ramos Santana La Burguesia Gaditana en la época Isabelina y en mi propia Tesis Doctoral, Gloria Espigado Tocino: La Primera República en Cádiz. Estructura social y comportamiento politico durante 1873. Caja de San Fernando, Sevilla y Jerez. Cádiz, 1993.

(19) "Lista definitiva ultimada con arreglo a lo dispuesto en el Art. 113 de la Ley de 18 de julio de 1865 , que comprende con sus nombres y apellidos paterno y materno, profesión y domicilio y contribución que pagan, a todos los Electores para Diputados a Cortes, pertenecientes al distrito Electoral de Cádiz". Boletín Oficial de la Provincia de Cádiz, enero de 1868. 
Si pasamos al segundo aspecto que aquí nos interesa, aquel que se refiere a los viajes que se hacen a su cargo, es evidente que por el número de barcos consignados a nombre de los hermanos Luciano y Horacio Alcón estamos ante la sociedad más importante de todas. Si éstos acaparan el 22,3\% del total de los encargos, lo que queda se reparte con relativa equidad entre los demás consignatarios: el comillano Antonio López sigue con un 6,6\%, detrás los Sicre con un 6,5\%, José Esteban Gómez y Juan Duncan Shaw se sitúan en torno al 5\%, así hasta el modesto 1\% de Manuel Amusátegui o Cesar Lowental.

En la familia Alcón hay un ex-diputado a Cortes, Horacio, dos cónsules, Aurelio que lo es de Italia y Ramón que lo es de Mónaco, al mismo tiempo que vicecónsul italiano. Todos ellos forman parte de la sociedad mercante "Luciano y Horacio Alcón", y sus barcos, vapores en 223 casos, transitan la Península, arribando desde Málaga en 87 ocasiones, Sevilla con 42 viajes y Gibraltar con 20 llegadas por el sur, y La Coruña, Vigo y Bayona, en el norte, con 23, 20 y 16 viajes respectivamente, saliendo en contadas ocasiones al extranjero. Probablemente los hermanos Alcón no son los armadores de todos los barcos que vienen consignados a sus nombres, en algunos casos actuarían como simples administradores en esta ciudad de otros navieros. Lo que decimos es fácil de comprobar cuando observamos que las llegadas de los vapores Ebro, Miño, Duero, Tajo y Francolí, propiedad de la Compañía Hispano-Inglesa que Pablo María Tintoré, uno de los armadores más importantes de la Ciudad Condal junto a Antonio López, tiene concertada con un consignatario de Liverpool, se dejan bajo su responsabilidad en una de las numerosas escalas que estos barcos efectúan en el trayecto que une al puerto catalán y la ciudad inglesa (20).

Siguiendo en la línea del cabotaje, el catalán Juan Buhigas se especializa en el uso del vapor entrando desde Vigo, Sevilla y Gibraltar, en cuyo último caso los barcos no son nacionales sino ingleses. José Esteban Gómez Peñasco, natural de Cádiz y cónsul de Portugal, también utiliza vapores de España dedicados al cabotaje de sus costas. Málaga, Sevilla y Vigo son los puertos más visitados, aunque sale al exterior en 10 de las 56 ocasiones. Los negocios del, también, gaditano Joaquín del Cubillo comprenden dos facetas desempeñadas siempre por vapores ingleses, o bien los barcos que consigna provienen de Málaga, vía Gibraltar, o bien su procedencia atañe a muelles ingleses como los de Londres; Cardiff, Dover, Glasgow o Shoreham. La familia

(20) Carlos LLORCA BAUS, Op. Cit., p. 73. 
Sobrino tiene ascendente gallego. Nacido en La Gutrdia es Benardino de Sobrino, que parece conservar de una pasada etapa indiana el honor de representar diplomáticamente a Guatemala. La Compañía de Vapores Sobrino, propiedad del cónsul, del cual conocemos por propio testimonio su vocación librecambista (21), contrata también vapores para circunnavegar el país entre Vigo y Málaga con los vapores Bayo, Duro, Arana y Cifuentes.

Otro capítulo de este transporte volcado hacia el comercio nacional y del que tan sólo nos quedaría por enumerar otros representantes de menor entidad como Antonio de Zulueta y Manuel Cadarso, viene de la mano de los vapores-correo que algún particular concierta con el Gobierno para mantener una ruta regular entre la Península y las posesiones ultramarinas. La familia Retortillo Imbrech toma a su cargo la línea de las Islas Canarias. Los tres hermanos, Jose María, conde de Torres y vizconde de Retortillo, Guillermo y Joaquín eran dueños de la sociedad "Retortillo Hnos.", encargada de enlazar con Tenerife dos veces al mes con los vapores "América" y "Africa". Aunque en el padrón no se declara contribución industrial alguna, ésta no debía ser baja en relación a la importancia de sus negocios. Sí, en cambio, se especifican sus impuestos como propietarios, y en modo alguno la cifra es despreciable (casi 3.000 pts en el caso de José María). Hay que tener en cuenta que la mayoría de estos hombres de negocios compatibilizaban su actividad comercial con su gestión como propietarios, y así consta en la declaración profesional que hacen a la administración municipal.

La otra empresa de vapores encargada de una ruta específica con concierto gubernamental es la de Antonio López, marqués de Comillas, que establece el puente marítimo entre España y las colonias antillanas. Hablar de lo que significa este hombre para Cádiz es reconstruir el pasado industrial de nuestra ciudad, ya que es habitual relacionar su nombre con la construcción del más grande astillero que tuvo el país a fines del pasado siglo. No es fácil, en cambio, obtener noticias ciertas de los primeros años de su biografia como hombre de negocios, ni siquiera personas que se vincularon profesionalmente a la Compañía Trasatlántica y que glosaron su historia dan como cierta la fecha de su nacimiento (22).

(21) Dada a conocer en su opúsculo La Revolución y La Hacienda. Al Excmo. Sr. Ministro de Hacienda. Cádiz, 30 de octubre de 1868.

(22) Las dos obras sobre las que se basa buena parte de la información relacionada con la empresa de Antonio López y su persona son: Jorge Marique de Lara, La Marina Mercante Ochocentista y el Puerto de Cádiz, Ediciones de la Caja de Ahorros de Cádiz, 1973, y Carlos Llorca Baus, La Compañia Trasatlántica en las Campañas de Ultramar, Ed. Ministerio de Defensa, Madrid, 1990. 
La fecha más probable es la de 1817 (23), y el lugar, la población de Comillas en la provincia de Santander. Al igual que muchos naturales de allí, emigra muy joven a Cádiz para convertirse en un "montañés", como aquí se llama popularmente a los que vienen de allá, mozo de un figón. La ciudad gaditana tan sólo es una escala para los propósitos del joven comillano que en 1830 embarca en esta ciudad rumbo a las "Américas" con el viejo empeño de hacer fortuna. Empleado de comercio, primero, hasta que funda su propia compañía, su suerte va cambiando rápidamente al llegar a constituir un cierto patrimonio que consolida casándose con la hija de un comerciante catalán, Luisa Brú. Permanecerá en Cuba, dedicándose al cabotaje de la Isla hasta que regresa a España en 1853, fijando su residencia en Barcelona (24). La fundación de la Compañía "Antonio López y Cía" se efectuó el 11 de enero de 1857 (25). Sin entrar en más detalles sobre la evolución de sus negocios, diremos que los años que siguen lo consagran como un gran naviero, éxito que corona en 1861 cuando consigue la concesión gubernamental de los correos antillanos. Haciendo uso ya de nuestra base de datos, los vapores que se encargan de esta singladura son el "Méndez Núñez", el "Isla de Cuba", el "Puerto Rico", el "Antonio López", el "España" y el "Comillas". Aparte de la correspondencia, los pasajeros, el tabaco y el azúcar, en este año salen los días 15 y 30 de cada mes, como figura en la propia propaganda de la Compañía, con los contingentes de tropa enviados a la guerra que se mantiene con los independentistas cubanos. Estos barcos también tocan los puertos de Barcelona y Santander, ciudad ésta última hacia donde se había desplazado por orden ministerial uno de los correos mensuales reglamentarios, medida que tanto disgustaba a los gaditanos. Otros vapores como el "Santander", el "Madrid", el "Alicante" y el "Pasajes" los tenía López para la circunnavegación peninsular, recalando en los puertos de Santander, La Coruña y Vigo, por el Atlántico, Barcelona, Málaga y Alicante, por el Mediterráneo. Finalmente, otro capítulo importante de los negocios de este santanderino lo proyecta hacia el exterior, al fletar barcos noruegos (5) e ingleses (18) para traer carbón desde Cardiff en

(23) Su expediente personal localizado en el Archivo del Senado la refiere al 14 de ábril de 1817. Carlos Llorca Baus, Op. Cit., p. 17.

(24) CARRERA PUJAL Jaime, La Economia de Cataluña en el siglo XIX. Marina Mercante, Puertos Carreteras y Ferrocarriles. Vol. IV, Bosett, Barcelona, 1961, p. 73.

(25) Una vez ha sido encontrada en el Archivo Histórico de Protocolos de Madrid la correspondiente acta notarial. Entre los fundadores, además de Antonio López están su hermano Claudio, Patricio Satrústegui y el marqués de Salamanca. Carlos Llorca, Op. Cit., p. 23. 
22 ocasiones y de Swansea en otra. Seguir la pista de este gran hombre de negocios es descubrir una trayectoria culminada por el éxito personal. En 1881 se crea, por fin, la Compañía Trasatlántica, y al mismo tiempo se va construyendo el astillero más grande de cuantos existían en el país y en Europa por aquel entonces, legado que dejará a su hijo Claudio López Brú.

Antonio López no es el único que mantiene relaciones comerciales con Inglaterra; pasando ya a los consignatarios que se especializan con alguna zona en particular del extranjero tendríamos que citar al vizcaíno de Algorta Manuel Amusategui, que haciendo uso de los vapores "Irurac-Bat", "Emiliano", "Buenaventura" y "Aurrerá", nos llega en siete ocasiones desde Liverpool. En otro apartado distinto se inscriben sus cinco viajes desde Manila, vía Port-Said, en las inmediaciones del canal de Suez.

Los citados hasta aquí hacen uso exclusivo de barcos de matrícula española; sin embargo, Juan Duncan Shaw y Daniel Mac-Pherson y los que siguen a continuación recurren preferentemente al pabellón extranjero, en este caso todos los barcos son ingleses. El primero de ellos está a cargo del consulado de Austria-Hungría y, aunque nació en Inglaterra, lleva residiendo en nuestra ciudad desde 1817, de manera que sus hijos, Eduardo, Guillermo y Jacobo son ya gaditanos. Su especialidad, siempre en barcos de vela, se centra en el bacalao, que trae en seis ocasiones desde Terranova, lo que está en estrecha relación con la llegada en lastre de 19 embarcaciones procedentes de puertos ingleses como Limerick, Falmouth, Plymouth, Dartsmouth etc., con el objeto probable de cargar sal para salir con rumbo hacia aquellas pesquerías. Málaga, Alicante y otros puntos del Mediterráneo son tocados de forma secundaria.

Los hermanos Daniel y Guillermo son copartícipes de la firma MacPherson, siendo el segundo cónsul de Grecia y vicecónsul de su majestad británica. Daniel es gaditano, y Guillermo declara ser natural de Inglaterra, sospechamos que nacido en Gibraltar, puesto que otros miembros de la familia proceden de allí. Esta debe ser la razón que lo vincula a una línea regular que mantienen con los vapores "Galicia", "Britania" y "Península" y que baja desde Málaga pasando por Gibraltar. Los Mac-Pherson son agentes de la famosa casa inglesa de comercio Lloyd's. Sus vapores ingleses vienen desde los puertos principales de Irlanda y de Inglaterra con carbón (12), haciendo escala algunos en Huelva para cargar mineral.

Finalmente, otros dos hermanos, Antonio y Luis Sicre, dan nombre a la sociedad que constituyen. Luis es francés, cónsul de los Países 
Bajos, pero Antonio, mucho más joven, es gaditano, por lo que es probable que la situación de residente del primero se remonte al menos cuarenta años. Aunque la bandera que domina en la relación de veleros que se les adjudican es la francesa, también hay un lugar para el pabellón inglés. Estos últimos son los que traen carbón desde Cardiff, Newcastle o Swansea (18 viajes). Desde Africa traen azúcar en 7 ocasiones y avellanas en 2, y desde Francia una treintena de veleros en lastre salidos de Fecamp, Marsella, Burdeos, Saint-Vaast, Saint-Malo o Nantes. Son galos todos los barcos consignados por el súbdito francés Eduardo Collet, que actúa como agente de la Línea Peninsular Francesa de Vapores, con embarcaciones fácilmente reconocibles por el nombre: "Ville du Havre", "Ville de Brest", "Ville de Cadix", "Ville de Lisbonne", "Ville de Malaga" y "Ville de Marselle", que recorren la ruta Marsella-Cádiz, tocando varios puntos en el Mediterráneo como Málaga y Gibraltar, o. El Havre-Cádiz, haciendo escala en puntos atlánticos como Vigo o Lisboa.

Otros dos nombres extranjeros de indiscutible vinculación con Italia son Odero y Remorino. Luis Odero Cuesta ha nacido en Génova y de allí proceden sus barcos, que son italianos en su totalidad, haciendo escala en Mahón o en Valencia. Excepcionalmente traen el famoso mármol italiano, pero lo normal es que vengan sin carga, con el propósito de llevar nuestra sal hacia el cono sur del continente americano. El otro apellido, Remorino Samarino, de clara resonancia italiana, corresponde a tres hermanos que, sin embargo, son gibraltareños, aunque no es difícil constatar sus ascendencia genovesa por parte materna. La relación de éstos con Italia es clara, puesto que son agentes de las Cías. de Seguros Italianos y Austriacos. Pedro, además, es agente del Registro Italiano de Génova. Sus viajes concertados están sujetos al mismo comentario realizado sobre Luis Odero; es decir, entrada en lastre desde Génova o regreso desde puertos americanos.

En el área americana destacan las entradas de los veleros estadounidenses de Antonio Bensusan (españolizado en Benasusan), cónsul de Honduras, que traen y distribuyen por las proximidades (Sevilla, Málaga, etc.) las duelas, el petróleo y el tabaco venidos de allí. José Morales Borrero visita asiduamente el puerto norteamericano de NewYork, pero no descarta incursiones a las colonias de Cuba y Puerto Rico. Las duelas, el café, el petróleo, el azúcar y los cueros son, en general, los productos importados a su nombre. Los negocios del riojano de Cameros Miguel Martínez de Pinillos se reservan el área americana, con cuatro viajes desde New-York y tres desde las inmediacio- 
nes caribeñas con los típicos frutos de la zona y las duelas y el petróleo norteamericano. No parece muy relevante la actividad comercial desarrollada ese año por el padre del que será fundador, andando el tiempo, de una importante compañía mercante, la Pinillos Izquierdo; nos referimos a Antonio Martínez de Pinillos, que por ahora es un joven de 30 años que vive en la residencia de su progenitor aprendiendo junto a él el oficio de comerciante.

Los franceses Pedro Luis Lacave y Juan Pablo Echecopar Jaureguiben forman sociedad y se distribuyen, por un lado, el comercio que contacta con los puertos norteamericanos de New-York, New Orleans y Boston, y aquel que lo hace con la zona del Báltico, desde Brevig, Lovisa, Copenhague y Helsinhfors. El objeto perseguido en ambos casos es el mismo, traer duelas y madera para la empresa de tonelería al por mayor y carros porteadores que les pertenece.

El Báltico también tiene sus incodicionales, estos son Gustavo Ricardo Lagergren y Alejandro T. Christophersen, cónsul de Noruega, Suecia y Dinamarca. El primero bajo bandera sueca y el segundo bajo la noruega, realizan singladuras desde la zona escandinava con base en Arendahl, Carlshamn, Calmar, Elseneur, Christiansund o Umea, para traer madera a todos los puertos importantes del Mediterráneo. Para finalizar, César Lowental, o Lovental, posee la representación de la Compañía Holandesa de Vapores. Este alemán, nacido en Hamburgo, se establece en nuestra ciudad en 1859 y compagina sus negocios con los cargos consulares de Paraguay y viceconsulares de los Países Bajos. Sus viajes parecen contagiarse de esta particular mezcolanza de datos personales y tocan los puertos más dispares: Coloforte, Livorno, Palermo y Génova, en Italia; Marsella, Londres, Swansea, Amsterdam y Lisboa. Por último, el cónsul del Imperio alemán, Ernesto Kropf, trafica con una compañía de vapores de aquel país con el "Neapel", el "Messina", el "Hamburg" y el "Lissabon", que tienen su base en Hamburgo.

No existe lógica posible para los viajes que conciertan algunos consignatarios, como es el caso del británico, cónsul de Turquía, Carlos Harrison Younguer, así como para los negocios de los hermanos gaditanos, pero de ascendencia inglesa e italiana indiscutible, Rudolph Robersi, que lo mismo cruzan el Atlántico, hacía Matanzas, La Habana, New-York o New Orleans, que se quedan en la Península caboteando sus costas, que van por bacalao a Terranova, o visitan puertos europeos como Hamburgo o Burdeos. 


\section{ANEXOS \\ CUADRO 3 \\ CONSIGNATARIOS DE BUQUES EN CADIZ DURANTE 1873}

\begin{tabular}{|c|c|c|c|c|c|c|c|c|c|}
\hline Nombre & A & $\mathrm{B}$ & C & $\mathrm{D}$ & $\mathrm{E}$ & F & $\mathrm{G}$ & $\mathrm{H}$ & I \\
\hline Alcón, Luciano & Cruz de la Madera 24 & $\mathrm{SF} / \mathrm{SC}$ & 45 & $\mathrm{C}$ & Cádiz & \multicolumn{2}{|l|}{ Comercio } & 2.100 & \\
\hline Alcón, Aurelio & Verónica 12 & Correo & 46 & C & Cádiz & \multicolumn{4}{|c|}{ Comercio, Cónsul de Italia } \\
\hline Alcón, Horacio & Aduana 4 & & & & & \multicolumn{4}{|c|}{ Comercio, Ex-diputado } \\
\hline 'Amusátegui', Manuel & S. Francisco 8 & $\mathrm{SF} / \mathrm{SC}$ & 42 & $\mathrm{C}$ & Algorta & \multicolumn{2}{|c|}{ Comercio } & \multicolumn{2}{|l|}{954} \\
\hline Bensusan, Antonio & \multicolumn{9}{|c|}{ S. Francisco 37, Duque de la Victoria 2; Cónsul de Honduras } \\
\hline Buhigas, Juan & Mina 7 & $\mathrm{SF} / \mathrm{SC}$ & 54 & $\mathrm{C}$ & Cataluña & \multicolumn{2}{|l|}{ Consignatario } & \multicolumn{2}{|l|}{662} \\
\hline Cadarso, Manuel & Baluarte 7 & & & & & & & & \\
\hline Colón, Cristóbal & Consulado Viejo 5 & $\mathrm{SF} / \mathrm{SC}$ & 50 & $\mathrm{C}$ & Cádiz & \multicolumn{2}{|l|}{ Comercio } & 265 & \\
\hline Collet, Eduardo & Cruz de la Madera 11 & $\mathrm{SF} / \mathrm{SC}$ & 47 & $\mathrm{C}$ & Francia & \multicolumn{2}{|l|}{ Comercio } & 1.145 & \\
\hline Christophersen, A. T. & Mina 3 & \multicolumn{8}{|c|}{ SF/SC Cónsul de Suecia, Noruega y Dinamarca } \\
\hline Cubillo, Joaquín del & Columela 36 & Correo & 67 & $S$ & Cádiz & \multicolumn{2}{|l|}{ Capitalista } & 2.252 & 3.2154 \\
\hline Echecopar, Juan Pablo & Mina 6 & $\mathrm{SF} / \mathrm{SC}$ & 81 & $\mathrm{C}$ & Libaunes & Comercio & 1.150 & 1.669 & \multirow[t]{2}{*}{6.0569} \\
\hline Gómez, José Esteban' & Murguía 35 & Cortes & 45 & $\mathrm{C}$ & Cádiz & Consignataric & 235 & 1.250 & \\
\hline Kropf, Ernesto & \multicolumn{9}{|c|}{ Calvario 4, cuatro torres 4, Cónsul de Alemania } \\
\hline \multirow[t]{2}{*}{ Lacave, Pedro Luis } & Ahumada 16-18 & $\mathrm{SF} / \mathrm{SC}$ & 55 & $\mathrm{~V}$ & Francia & \multirow[t]{2}{*}{ Comercio } & 615 & & \multirow{2}{*}{$\begin{array}{l}3.2428 \\
6377\end{array}$} \\
\hline & & \multicolumn{4}{|c|}{ Sociedad Lacave, 3 socios } & & 1211 & 3830 & \\
\hline Lagergren, Gustavo Ricardo & S. Agustín 3 & Correo & 38 & $\mathrm{~S}$ & Suecia & \multicolumn{4}{|l|}{ Comercio } \\
\hline López, Antonio & Isabel la Católica 3 & $\mathrm{SF} / \mathrm{SC}$ & 56 & C & Comillas & \multicolumn{4}{|l|}{ Comercio } \\
\hline Lovental, César & Murguía 21 & Cortes & 37 & $S$ & Hamburgo & \multicolumn{4}{|l|}{ Comercio } \\
\hline Mac-Pherson, Daniel & S. Gines 4 & $\mathrm{SF} / \mathrm{SC}$ & 52 & S & Cádiz & & & & \\
\hline Mac-Pherson, Guillermo & Torrijos 2 & $\mathrm{SF} / \mathrm{SC}$ & 48 & $\mathrm{C}$ & Inglaterra & \multicolumn{4}{|l|}{ Comer/Propie } \\
\hline Martínez Pinillos, Miguel & Alameda 24 & $\mathrm{SF} / \mathrm{SC}$ & 68 & $\mathrm{~V}$ & Cameros & Comercio & 192 & 2.250 & \\
\hline Morales Borrero, José & Baluarte 12 & $\mathrm{SF} / \mathrm{SC}$ & 34 & $\mathrm{C}$ & Cádiz & Comercio & & 1.575 & \\
\hline Odero Cuesta, Luis & Gamonales 2 & $\mathrm{SF} / \mathrm{SC}$ & 41 & $\mathrm{C}$ & Génova & Comercio & & 954 & \\
\hline Remorino, Domingo & & & & & & & & & \\
\hline Remorino, Juan & Palna, 3 & Pópulo & 56 & $\mathrm{C}$ & Gibraltar & Consignataric & & & \\
\hline Remorino, Pédro & Argantonio 2 & Correo & 47 & $S$ & Gibraitar & Consignataric & & 637 & \\
\hline Retortillo, Guillermo & Camino 12 & $\mathrm{SF} / \mathrm{SC}$ & 56 & $\mathrm{C}$ & Câdiz & Comercio & 1.257 & & \\
\hline Retortillo, Joaquîn & Isabel la Católica & $\mathrm{SF} / \mathrm{SC}$ & & & & Comercio & 780 & & \\
\hline Retortillo, José María & Ancha 16 & Cortes & 59 & C & Cádiz & Comer/Propi & 2.795 & & \\
\hline Rudolph, Guillermo & Baluatte 14 & $\mathrm{SF} / \mathrm{SC}$ & & & & Comercio & & & \\
\hline Rudolph, Pedro & Cruz de la Madera 10 & $\mathrm{SF} / \mathrm{SC}$ & 54 & $\mathrm{C}$ & Gádiz & Comercio & 325 & 682 & 1.3503 \\
\hline Rudolph, Carlos & Baluarte 14 & $\mathrm{SF} / \mathrm{SC}$ & 60 & $S$ & Cádiz & Comercio & 1.342 & 682 & 3.3005 \\
\hline Shaw, Juan Duncan & Isabel la Católica 11 & $\mathrm{SF} / \mathrm{SC}$ & 71 & $\mathrm{~V}$ & Inglaterra & Comercio & & 1.550 & \\
\hline Sicre García, Antonio & Linares 18 & Constitución & 41 & C & Cádiz & Comercio & & 950 & \\
\hline Sicre Gellos, Luis & Baluarte 5 & SF/SC & 62 & $\mathrm{C}$ & Francia & Comercio & & 950 & \\
\hline Sobrino Vicente, Bernardino & Linares 17 & Constitución & 50 & C & Galicia & Comercio & 1.350 & & 7.0003 \\
\hline Sobrino Vicente, Ramón & Aduana 14-15 & Correo & 63 & $\mathrm{~V}$ & Galicia & Comercio & 1.062 & & \\
\hline Sobrino Vicente, Ricardo & Aduana 14-15 & Correo & 33 & $\mathrm{C}$ & Cádiż & Comercio & & 821 & \\
\hline Sobrino Vicente, Antonio & Aduana 14-15 & Correo & 26 & $S$ & Cádiz & Comercio & & 821 & \\
\hline Younguer, Carlos Harrison & Aduana 16. & Correo & 49 & $\mathrm{C}$ & Inglaterra & Consignataric & Consul & de Tur & quía \\
\hline Zulueta, Antonio de & P. Nieves 3 & Pópulo & 57 & C & Cadiz & Consignataric & 427 & 662 & 9.700 \\
\hline
\end{tabular}
A. Domicilio
B. Barrio
C. Edad
D. Estado Civil: S, soltero; C, casado, y V, viudo
E. Lugar de nacimiento
F. Profesión
G. Contribución Territorial en pts.
H. Contribución Industrial en pts.
I. Haberes anuales en pts.
J. Servicio doméstico 


\section{CUADRO 4 \\ CARGAS QUE DECLARAN LOS BUQUES ENTRADOS EN EL PUERTO}

\begin{tabular}{|c|c|c|c|}
\hline LASTRE. & CUEROS ...... & PERAS ...................4 & ALMAGRA ......... \\
\hline GENERAL. & BACALAO ............ & CASTAÑAS ..............4 & FIDEOS .............. \\
\hline CARBON ..................... & HIERRO $\ldots . . . \ldots \ldots \ldots \ldots . . . . .14$ & ACEITE ................... 3 & ALGODON .......... \\
\hline VINO ......................204 & BATATAS ...............14 & GARBANZOS ...........3 & LINAZA .............. \\
\hline VARIOS EFECTOS & TABACO ................13 & AVELLANAS ..............3 & VERDURAS ....... \\
\hline SARDINAS ................156 & AGUARDIENTE ........12 & ARROZ ..................... 3 & VIDRIOS .............. \\
\hline PESCADO .................113 & MAIZ ......................12 & HABICHUELAS .. & HUESOS ............. \\
\hline FRUTA .............................. & LOSAS ….................12 & BOTAS VACIAS ........3 & RON $\ldots \ldots \ldots \ldots$ \\
\hline LADRILLOS ….............72 & 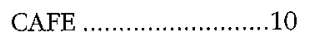 & PAJA & ALUMBRE ...... \\
\hline HUEVOS ....................72 & 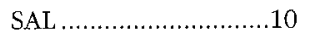 & HABAS...... & FUSILES $\ldots \ldots \ldots \ldots \ldots \ldots 1$ \\
\hline DUELAS .................... 59 & CORRESPONDENCIA..10 & MARMOL ................ 3 & TOMIZA DE PALMA ...1 \\
\hline MADERA ….................59 & GALLINAS .................. 8 & POLVORA ................ & ALGARROBAS ......... \\
\hline CEBADA .................46 & ACEITUNAS . ............. 8 & LOMO ............... & SOGAS .................. \\
\hline HIGOS ...................,46 & TUBERIAS ................... 8 & SALITRE................ 2 & VARILLA ............... \\
\hline NARANJAS ..................44 & ATUN $\ldots \ldots \ldots \ldots \ldots \ldots \ldots \ldots \ldots \ldots \ldots \ldots \ldots \ldots$ & ADOQUINES ...........2 & GUANO ............. \\
\hline FRUTOS ..................... & ESPIRITU ................6 & RECREO $\ldots \ldots \ldots \ldots \ldots \ldots . . .2$ & BARRO $\ldots \ldots \ldots \ldots \ldots$ \\
\hline ESPARTO ...................32 & PIPAS VACIAS ...........6 & SANGUIJUELAS ........2 & OREGANO............. \\
\hline CERDOS ......................... & CAL_............................... & JUNCOS $\ldots \ldots \ldots \ldots \ldots \ldots . . . . .2$ & UVAS .................. \\
\hline AZUCAR ..................... 30 & PAPEL $\ldots \ldots \ldots \ldots \ldots \ldots \ldots$ & HIELO $\ldots \ldots \ldots \ldots \ldots \ldots \ldots . . . \ldots$ & TEJIDOS ............... \\
\hline CHACINA .................... & MINERAL ...................6 & CAÑAS .................. & ARROPE .............. 1 \\
\hline TRIGO ......................23 & CORCHO .................. 5 & TABLAS ................... 2 & PIEDRAS DE MOLINO...1 \\
\hline PAPAS .......................23 & PASAJEROS ................. 5 & DROGAS $\ldots \ldots \ldots \ldots \ldots . . .2$ & ASTAS .................. 1 \\
\hline PETROLEO $\ldots \ldots \ldots \ldots \ldots \ldots . . . . . . .20$ & COBRE .................... 5 & CANTERIA ................ 2 & TEJAS ................. \\
\hline 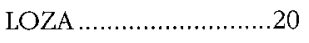 & MOJAMA ................ & HARTNA ................. 2 & $\mathrm{CACAO}, \ldots \ldots \ldots \ldots$ \\
\hline JABON .....................19 & TRAVIESAS ............... & PIPAS DE ATUN ......2 & PASAS $\ldots \ldots \ldots$ \\
\hline
\end{tabular}

\section{CARGAS QUE DECLARAN LOS BUQUES SAIIDOS DEL PUERTO}

\begin{tabular}{|c|c|c|c|}
\hline SAL .......................... 440 & 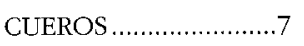 & ESPARTO ............ & \\
\hline VINO …...................177 & PLOMO ...................... & PAPEL ................ & $\mathrm{F}$ \\
\hline ENERAL ... & TRIGO $\ldots \ldots \ldots$ & EBADA. & QUESO . \\
\hline S ....108 & $\ldots .6$ & ALITRE. & HIELO ... \\
\hline$\ldots \ldots .82$ & TABACO ...... & $\ldots 2$ & ARROZ.. \\
\hline ...........81 & AGI & ACEITUNAS . & AVELLAI \\
\hline ............32 & $\ldots \ldots .5$ & TABI & RECREO... \\
\hline ...19 & & .2 & \\
\hline$\ldots .14$ & & POLVOR & NAR \\
\hline .....14 & $\ldots .4$ & ALGODO & HUESOS ..... \\
\hline$\ldots .12$ & & & RAII \\
\hline .11 & & JABON ... & FIDEOS $\ldots$ \\
\hline$\ldots . .9$ & & PIPERTA .... & HARINA.. \\
\hline CALAO & MTNERAL...... & $A D A T A S$ & \\
\hline
\end{tabular}




\section{CUADRO 5 \\ RELACION DE PUERTOS EN CONTACTO CON EL DE CADIZ EN 1873}

\begin{tabular}{|c|c|c|c|c|c|}
\hline & ENTRADAS & SALIDAS & ENTl & RADAS & SALIDAS \\
\hline AMERICA DEL NORTE & 59 & 214 & & & \\
\hline TERRANOVA & 6 & 139 & BOSTON & 5 & 17 \\
\hline GASPE & 1 & 4 & NEW YORK & 37 & 25 \\
\hline SHIPPEGAN & 1 & & PHILADELPHIA & 1 & 4 \\
\hline QUEBEC & 1 & & NEW ORLEANS & 7 & 1 \\
\hline BELLE ISLE & & 1 & PENSACOLA & & 1 \\
\hline S. PIERRE ET MIQUELON & & 6 & PORTLAND & & 2 \\
\hline HALIFAX & & 4 & GLOUCESTER & & 9 \\
\hline MONTREAL & & 1 & & & \\
\hline AMERICA DEL SUR & 63 & 203 & & & \\
\hline LA HABANA & 33. & 43 & BAHIA & & 4 \\
\hline MATANZAS & 6 & & RIO DE JANEIRO & & 3 \\
\hline CUBA & 2 & & RIO GRANDE & 2 & 25 \\
\hline SANTIAGO & & 3 & MONTEVIDEO & & 27 \\
\hline PUERTO RICO & 6 & 20 & RIO DE LA PLATA & & 44 \\
\hline MAYAQUEZ & 3 & & FRAY VENTOS & & 2 \\
\hline VERACRUZ & 1 & 12 & BUENOS AIRES & 8 & 17 \\
\hline TAMPICO & & 1 & IQUEQUE & 2 & \\
\hline MATAMOROS & & 2 & & & \\
\hline ASIA & 11 & 14 & & & \\
\hline BOMBAY & 1 & & MANILA & 9 & 10 \\
\hline SINGAPUR & 1 & 4 & & & \\
\hline AFRICA & 111 & 7 & & & \\
\hline ZANZIBAR & 1 & & LARACHE & 12 & 2 \\
\hline REUNION & 6 & 1 & SAFFI & & 3 \\
\hline ISLA MAURICIO & 3 & & CABO VERDE & & 1 \\
\hline TANGER & 57 & & MADEIRA & 1 & \\
\hline TETUAN & 14 & & FUNCHAL & 11 & \\
\hline MAZAGAN & 2 & & SIERRA LEONA & 1 & \\
\hline MOGADOR & 2 & & RIO MUNI & 1 & \\
\hline CHAFARINAS & & 1 & & & \\
\hline EUROPA & 514 & 428 & & & \\
\hline GRAN BRETAÑA & 193 & 166 & & & \\
\hline GLASGOW & 10 & 14 & DARTSMOUTH & & \\
\hline LEITH & & 6 & EXETER & & 1 \\
\hline NEWCASTLE & 25 & 2 & PLYMOUTH & 3 & 2 \\
\hline SUNDERLAND & 5 & & FAIMOUTH & 3 & 2 \\
\hline BARROW & 1 & & JERSEY & 3 & 3 \\
\hline LIVERPOOL & 28 & 27 & SCILLY & 1 & \\
\hline HULL & & 4 & BRISTOL & 3 & 7 \\
\hline
\end{tabular}




\begin{tabular}{|c|c|c|c|c|c|}
\hline GOOLE & 1 & & NEWPORT & 3 & \\
\hline LONDRES & 7 & 84 & CARDIFF & 68 & \\
\hline ROCHESTER & & 1 & SWANSEA & 19 & 1 \\
\hline NEW HAVEN & & 1 & MILFORD & 1 & \\
\hline DOVER & 1 & & BELFAST & & 2 \\
\hline COWES & 2 & & & & \\
\hline POOLE & 1 & & & & \\
\hline IRLANDA & 7 & 11 & & & \\
\hline DUBLIN & 6 & 8 & CORK & & 2 \\
\hline LIMERIK & 1 & & & & \\
\hline FRANCIA & 66 & 89 & & & \\
\hline NIZA & 3 & & SAINT-MALO & 3 & 1 \\
\hline SEYNE & 1 & & GRANVILLE & 3 & \\
\hline MARSELLA & 23 & 66 & SAINT-VAAST & 2 & \\
\hline BOURDEOS & 7 & 3 & LE HAVRE & 7 & 14 \\
\hline LA ROCHELLE & 1 & & TROUVILLE & 1 & \\
\hline NANTES & 3 & 1 & FECAMP & 7 & \\
\hline S. NAZAIRE & 2 & 4 & DUNKERQUE & 3 & \\
\hline ITALIA & 63 & 10 & & & \\
\hline GENOVA & 48 & 5 & SICILIA & & 1 \\
\hline LIORNA & 3 & 2 & PALERMO & 5 & \\
\hline CIVITA VECCHIA & 2 & & MESSINA & 3 & \\
\hline NAPOLES & 1 & 1 & CARLOFORTE & 1 & \\
\hline BARI & & 1 & & & \\
\hline BELGICA & 10 & 2 & & & \\
\hline AMBERES & 10 & 2 & & & \\
\hline HOLANDA & & 10 & & & \\
\hline ULARDINGEN & & 2 & AMSTERDAM & & 8 \\
\hline ALEMANIA & 14 & 6 & & & \\
\hline HAMBURGO & 14 & 6 & & & \\
\hline DINAMARCA & 1 & 12 & & & \\
\hline COPENHAGUE & 1 & 10 & FREDRISKHAN & & 2 \\
\hline NORUEGA & 8 & 18 & & & \\
\hline CRHISTIANSUND & 5 & 11 & GRIMSTAN & 1 & \\
\hline BERGEN & 1 & 7 & ARENDHAI, & 1 & \\
\hline SUECIA & 10 & 2 & & & \\
\hline HELSINFOR & 6 & & CALMAR & 1 & \\
\hline CARLSHAMN & 2 & 2 & UMEA & 1 & \\
\hline
\end{tabular}




\begin{tabular}{|c|c|c|c|c|c|}
\hline FINLANDIA & 6 & 1 & & & \\
\hline LOVISA & 5 & & BORGA & 1 & 1 \\
\hline RUSIA & & 6 & & & \\
\hline S. PETERSBURGO & & 5 & RTGA & & 1 \\
\hline PORTUGAL & 136 & 95 & & & \\
\hline VIANNA & 2 & 2 & SETUBAL & & 8 \\
\hline OPORTO & 7 & 3 & OLHAO & 40 & 8 \\
\hline LISBOA & 29 & 82 & FUSETA & 58 & \\
\hline ESPAÑA & 1.787 & 753 & & & \\
\hline ROSAS & & 2 & MOGUER & 135 & 1 \\
\hline MATARO & 1 & & CARTAYA & 95 & \\
\hline BARCELONA & 31 & 86 & HUELVA & 103 & 34 \\
\hline VENDRELL & 4 & & ISLA CRISTINA & 133 & 1 \\
\hline TARRAGONA & ' 18 & 2 & AYAMONTE & 98 & 2 \\
\hline S. CARLOS DE LA RAPITA & 3 & & HIGUERITA & 11 & 2 \\
\hline VINAROZ & 6 & & CANARLAS & & 18 \\
\hline ANDRAIX & 6 & & LAS PALMAS & 2 & 14 \\
\hline PALMA & 3 & 16 & TENERIFE & 22 & 15 \\
\hline MAHON & & 1 & LA GUARDIA & 2 & 1 \\
\hline IBIZA & 1 & & BAYONA & 22 & 5 \\
\hline VALENCIA & 21 & 9 & EL CARRIL & & 2 \\
\hline DENIA & 1 & 1 & VIGO & 122 & 90 \\
\hline ALICANTE & 42 & 17 & MARIN & 4 & 4 \\
\hline SANTA POLA & 3 & & VILLAGARCIA & 6 & 1 \\
\hline TORREVIEJA & 3 & & NOYA & & 1 \\
\hline CARTAGENA & 6 & 4 & MUROS & 10 & 1 \\
\hline AGUILAS & 2 & & CORCUBION & & 1 \\
\hline ALMERIA & 41 & & P. DEL CARAMIÑA & L 1 & \\
\hline ADRA & 9 & 3 & LA CORUÑA & 24 & 5 \\
\hline ALBUÑOL & 1 & & BETANZOS & & 3 \\
\hline MOTRIL & 3 & 1 & FERROL & 3 & 1 \\
\hline SALOBREÑA & 4 & & ORTIGUEIRA & 1 & \\
\hline ALMUÑECAR & 5 & & VIVERO & & 1 \\
\hline NERJA & 6 & & RIBADEO & 1 & \\
\hline VELEZ-MALAGA & 2 & 2 & LUARCA & 1 & 2 \\
\hline MALAGA & 307 & 62 & AVILES & 2 & \\
\hline FUENGIROLA & 1 & & GIJON & 13 & 12 \\
\hline ESTEPONA & 15 & & RIBADESELLA & 1 & 1 \\
\hline GIBRALTAR & 22 & 99 & SANTANDER & 15 & 58 \\
\hline ALGECIRAS & 173 & 68 & BILBAO & 2 & 39 \\
\hline TARIFA & 86 & 34 & ZUMAYA & 3 & 2 \\
\hline CEUTA & 7 & & S. SEBASTIAN & 4 & 5 \\
\hline SANLUCAR & 30 & & FUENTERRABIA & & 10 \\
\hline BONANZA & 87 & 2 & PASAJES & 1 & \\
\hline SEVILLA & 307 & 188 & & & \\
\hline
\end{tabular}

